\title{
A New Chaotic System with Only Nonhyperbolic Equilibrium Points: Dynamics and Its Engineering Application
}

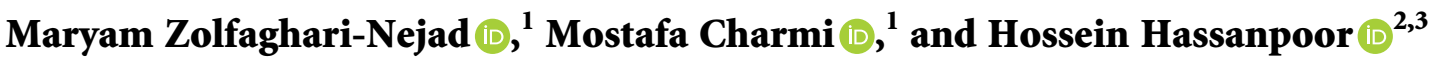 \\ ${ }^{1}$ Department of Electrical Engineering, Faculty of Engineering, University of Zanjan, Zanjan, Iran \\ ${ }^{2}$ Department of Cognitive Sciences, Dade Pardazi Shenakht Mehvare Atynegar (DSA) Institute, Tehran, Iran \\ ${ }^{3}$ Department of Medical Engineering, Energy Institute of Higher Education, Saveh, Iran \\ Correspondence should be addressed to Mostafa Charmi; charmi.mostafa@znu.ac.ir
}

Received 25 August 2021; Revised 14 November 2021; Accepted 13 December 2021; Published 12 January 2022

Guest Editor: Viet-Thanh Pham

Copyright (C) 2022 Maryam Zolfaghari-Nejad et al. This is an open access article distributed under the Creative Commons Attribution License, which permits unrestricted use, distribution, and reproduction in any medium, provided the original work is properly cited.

\begin{abstract}
In this work, we introduce a new non-Shilnikov chaotic system with an infinite number of nonhyperbolic equilibrium points. The proposed system does not have any linear term, and it is worth noting that the new system has one equilibrium point with triple zero eigenvalues at the origin. Also, the novel system has an infinite number of equilibrium points with double zero eigenvalues that are located on the $z$-axis. Numerical analysis of the system reveals many strong dynamics. The new system exhibits multistability and antimonotonicity. Multistability implies the coexistence of many periodic, limit cycle, and chaotic attractors under different initial values. Also, bifurcation analysis of the system shows interesting phenomena such as periodic window, period-doubling route to chaos, and inverse period-doubling bifurcations. Moreover, the complexity of the system is analyzed by computing spectral entropy. The spectral entropy distribution under different initial values is very scattered and shows that the new system has numerous multiple attractors. Finally, chaos-based encoding/decoding algorithms for secure data transmission are developed by designing a state chain diagram, which indicates the applicability of the new chaotic system.
\end{abstract}

\section{Introduction}

In recent decades, particular attention has been paid to the chaos that arises in nonlinear dynamic systems. This is due to various potential applications of chaotic systems in science and engineering fields. With special attention accorded to chaotic dynamics, many researchers strive to develop new chaotic systems with interesting properties and complex behaviors SPROTT [1]. Recently, some chaotic systems are reported with different characteristics of equilibrium points Sprott [2], with no equilibria Zhang et al. [3], only stable equilibrium point Deng et al. [4], with different shapes of equilibria Pham et al. [5], and with a line equilibrium Jafari and Sprott [6].

The equilibrium point of the system is defined as the simplest possible solution of its differential equation $\dot{x}(t)=f((x))=0$. The equilibrium points could be hyperbolic or nonhyperbolic. If all eigenvalues of the corresponding Jacobian matrix have nonzero real parts, the equilibrium of the system is hyperbolic. In these systems, the existence of chaos is proved by using the Shilnikov theorem Lawande and Maiti [11]. According to the Shilnikov theorem, a system must have at least one equilibrium point of saddle-focus type with three eigenvalues $\gamma, \delta \pm i \omega$ that should satisfy $\gamma \delta<1$, $|\gamma|>|\delta|>0$, and $\omega \neq 0$. Suppose that there are homoclinic/ heteroclinic orbits; then, chaos can be shown to exist (Kingni et al. [12]; Leonov [13]). In contrast, if at least one of the real parts of the corresponding eigenvalues is zero, the equilibrium is nonhyperbolic Izhikevich [14]. So far, a few chaotic systems with nonhyperbolic equilibrium points, namely, nonShilnikov chaotic systems, are formulated and studied (Li and Xiong [10]; Cai et al. [8]; Singh and Roy [15]). For example, a new Lorenz-like chaotic system with two nonhyperbolic equilibrium points of the type double zero eigenvalues is introduced in Kamdem Kuate et al. [7]. Also, a grid multiwing chaotic system with nonhyperbolic equilibria is investigated in Zhang et al. [3]. In Chen et al. [9], a 3D chaotic system with only one equilibrium point at the origin is proposed that has three zero eigenvalues. The reported non-Shilnikov chaotic 
systems with various nature of equilibrium points and several types of nonlinearities are given in Table 1. To the best of our knowledge, there has been little research on nonhyperbolic equilibria with triple or double zero eigenvalues in dynamical systems.

In the investigation of chaos theory, it is important to design new chaotic systems with some interesting phenomena such as period-subtracting phenomenon by Zolfaghari-Nejad et al. [16], multistability Dong et al. [17], antimonotonicity Liu and $\mathrm{Iu}$ [18]; the phenomena of asymmetric bistability by $\mathrm{Li}$ et al. [19]; hidden chaotic attractor by Jahanshahi et al. [20]; and some features related to their equilibria. A system is called multistable if there are different coexisting attractors in the constant parameters and various initial conditions by Jafari and Kapitaniak [21]. It is generally believed that the system with coexisting attractors has better flexibility and plasticity in performance (Lai et al. [22]). In Li and Sprott [23], Pisarchik and Grebogi [24], and $\mathrm{Li}$ et al. [25], the authors gave a comprehensive analysis of the coexisting attractors in Lorenz systems. In Lai et al. [22], the authors consider creating a new autonomous chaotic system with many coexisting attractors. The system in Lai et al. [22] generates multiple coexisting attractors by increasing the number of equilibrium points, but no application is mentioned for them. It is well recognized from different works that multiple coexisting attractors depend on the number of equilibrium points. As a result, some periodic functions like sine can be used to design chaotic systems with multiple coexisting attractors such as Lai et al. [26] and Li et al. [25].

The phenomenon of antimonotonicity is observed in various nonlinear systems such as Kengne et al. [27], Kingni et al. [28], and Signing et al. [29]. In this phenomenon, periodic orbits can be yielded from period-doubling bifurcation and then annihilated via reverse period-doubling as a control parameter is slowly altered.

In general, chaotic systems have a linear term in addition to a nonlinear one Kengne et al. [30]. Chaotic systems without linear terms have rarely been investigated in the literature Zhang et al. [31], Xu and Wang [32], Pham et al. [33], and Vaidyanathan [34]. A simple chaotic system without linear terms is proposed in Pham et al. [35]. Furthermore, a chaotic system with only quadratic and constant terms is introduced in Mobayen et al. [36].

A chaotic system is used in many engineering applications in Guanrong [37]. The most valuable application is secure communication. During the past few years, many chaotic substitution boxes (S-box) have been proposed such as Çavuşoğlu et al. [38], Wang et al. [39], García-Guerrero et al. [40], and Ullah et al. [41]. S-box is the most basic unit in encryption algorithms (Mobayen et al. [42]). On the other hand, some applications have used chaotic systems to achieve secure data transmission as in Gan et al. [43], Tirandaz and Karami-Mollaee [44], Dhall et al. [45], and Nesa et al. [46] and image encryption as in Shaukat et al. [47]. Data confidentiality is obligatory during transmission, especially in the military, medical, and financial applications (Noura et al. [48]). In this work, we propose chaos-based encoding/decoding algorithms for a secure data transmission scheme using the novel chaotic system.
In this paper, a new three-dimensional chaotic system with only nonhyperbolic equilibrium points is introduced. The main characteristics of the system can be summarized as follows: (i) the novel system has triple zero eigenvalues at the origin; (ii) the system has many equilibria with double zero eigenvalues; (iii) the chaotic system does not have any linear term; (iv) the new system has no reflection symmetry property about any axis; (v) the phenomenon of infinite coexistence of multiple periodic, limit cycle, and chaotic attractors is observed; (vi) this system can exhibit antimonotonicity phenomena such as chaotic bubbles and period-2 bubbles; (v) it has very scattered spectral entropy (SE) distribution under different initial values. Furthermore, we address chaos-based encoding and decoding algorithms for a secure data transmission scheme by designing a state chain (SC) diagram, which indicates the applicability of the new chaotic system.

The rest of this paper is organized as follows. In Section 2, we describe the chaotic mathematical model and analyze the equilibrium points. In Section 3, we will show the nonlinear characteristics of the system in terms of bifurcation diagrams, Lyapunov exponents (LEs) spectrum, phase portrait, and spectral entropy (SE) distributions. In Section 4, we describe the details of the engineering application of the proposed system comprising chaos-based encoding/decoding algorithms for a secure data transmission scheme. The performance of this scheme is analyzed in Section 5. Finally, the conclusion of the current research work is summarized in Section 6.

\section{Description and Analysis of the Proposed System}

2.1. The System Model. The structure of the proposed system is as follows:

$$
\begin{aligned}
& \dot{x}=-p_{1} x^{3}+p_{2} y^{3}+p_{3} x z^{2}, \\
& \dot{y}=p_{4} \sin (x y-z)-p_{5} x^{3}, \\
& \dot{z}=p_{6} \sin (x y z)+p_{7} \sin (x)
\end{aligned}
$$

where $(x, y, z)$ are state variables and $p_{i}(i=1,2, \ldots, 7)$ are (real) positive parameters of the system. System (1) is a thirdorder autonomous with seven nonlinearity terms. Notice that the nonlinearity terms are either sine or cubic. It is clearly seen the chaotic system does not have any linear or constant term. System (1) has no reflection symmetry property about any axis, which indicates the system could not have bistability or symmetric attractors.

When the parameters are set to $\left(p_{1}, p_{2}, p_{3}, p_{4}\right.$, $\left.p_{5}, p_{6}, p_{7}\right)=(3.964,7,7,5,4.5,2.9,1)$ and the initial value is set as $\left(x_{0}, y_{0}, z_{0}\right)=(-1,-1,-1)$, the proposed system generates a chaotic attractor. In this case, the three Lyapunov exponents are calculated as $\mathrm{LE}_{1}=0.2603, \mathrm{LE}_{2}=0.002$, and $\mathrm{LE}_{3}=-5.491$ (by using ode45 numerical solver and the simulation time $T=1000 \mathrm{~s})$. At this initial value, the fractional Kaplan-York dimension is $D_{\mathrm{KY}}=2+\left(\left(\mathrm{LE}_{1}+\mathrm{LE}_{2}\right)\right)$ / $\left(\left|\mathrm{LE}_{3}\right|\right)=2.0478$, which confirms the chaotic behavior $\left(D_{\mathrm{KY}}>2\right)$. Figure 1 shows the corresponding phase portraits of this strange attractor in $x-y, x-z$, and $y-z$ planes, from left to right, respectively. 
TABLE 1: Type of nonlinearities in non-Shilnikov chaotic systems with various nature of their equilibria.

\begin{tabular}{lcc}
\hline Reference of papers & Type of nonlinearities & Nature/number of equilibrium points \\
\hline Kamdem Kuate et al. [7] & Absolute value and signum & Double zero eigenvalues/2 \\
Cai et al. [8] & Quadratic and absolute value & Quadratic \\
Chen et al. [9] & Quadratic and exponential & One pair of pure-imaginary eigenvalues/Infinite \\
Li and Xiong [10] & Sine and cubic & Triple zero eigenvalues/1 \\
This work & One pair of pure-imaginary eigenvalues/2 \\
\hline
\end{tabular}

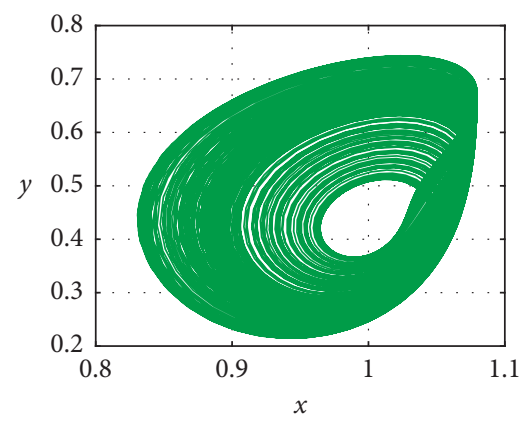

(a)

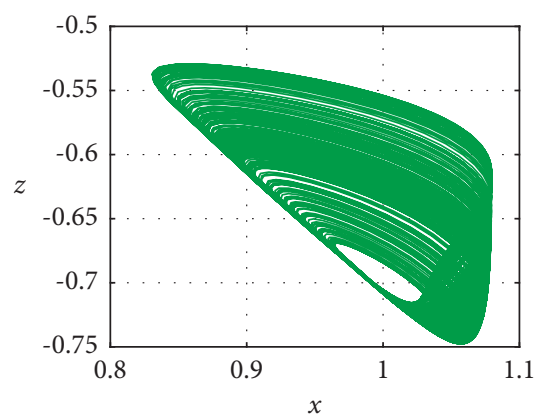

(b)

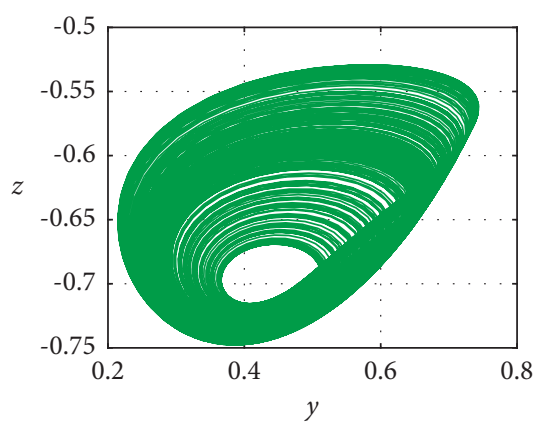

(c)

Figure 1: Phase portraits of the system with $\left(p_{1}, p_{2}, p_{3}, p_{4}, p_{5}, p_{6}, p_{7}\right)=(3.964,7,7,5,4.5,2.9,1)$ and initial value $(-1,-1,-1)$ projected on (a) $x-y$ plane, (b) $x-z$ plane, and (c) $y-z$ plane.

2.2. Analysis of Equilibrium Points. Suppose that $P\left(x_{*}, y_{*}, z_{*}\right)$ is the equilibrium point of the system (1); then,

$$
\begin{aligned}
-p_{1}\left(x_{*}\right)^{3}+p_{2}\left(y_{*}\right)^{3}+p_{3} x_{*}\left(z_{*}\right)^{2} & =0, \\
p_{4} \sin \left(x_{*} y_{*}-z_{*}\right)-p_{5}\left(x_{*}\right)^{3} & =0, \\
p_{6} \sin \left(x_{*} y_{*} z_{*}\right)+p_{7} \sin \left(x_{*}\right) & =0 .
\end{aligned}
$$

We have a system with nonlinear equations and three unknowns. Nonlinear equations cannot be solved analytically in general. We used Newton's method for solving this system, which involves using the Jacobian matrix. Newton's method is one of the most popular numerical methods and is referred to in Courtney [49]. By solving equation (2), we have $x_{*}=y_{*}=0$ and $z_{*}=k \pi(k=0, \pm 1, \pm 2, \pm 3, \pm 4, \ldots)$. It is clear that one equilibrium point is located at the origin, and the others are located on the $z$-axis. The Jacobian matrix is obtained by the linearizing system $(1)$ at $P\left(x_{*}, y_{*}, z_{*}\right)$ as

$$
J=\left[\begin{array}{ccc}
p_{3} z_{*}^{2} & 0 & 0 \\
0 & 0 & -p_{4} \cos \left(z_{*}\right) \\
p_{7} & 0 & 0
\end{array}\right] .
$$

The characteristic equation of $J$ is

$$
\lambda^{3}-p_{3}\left(z_{*}\right)^{2} \lambda^{2}=0
$$

Equation (4) has triple zero eigenvalues in its equilibrium point at the origin. This equilibrium point is nonhyperbolic and characterized by a $3 \mathrm{D}$ center manifold. Also, the corresponding eigenspace gives rise to a slow manifold. In Jiang et al. [50], Gamero et al. [51], and Freire et al. [52], some methods have been suggested to derive the normal forms on the center manifold, which can be used to study the dynamics near triple zero eigenvalues. The stability of this equilibrium point cannot be determined under the Shilnikov criteria. So, the stability of this case is investigated numerically by tracking the final state of the system with initial values around the equilibrium point (Faghani et al. [53]). The result of this investigation shows that the origin is unstable.

The characteristic equation (4) indicates that the Jacobian matrix (3) has double zero eigenvalues $\left(\lambda_{1,2}=0\right)$ and one nonzero eigenvalue $\lambda_{3}=p_{3} k^{2} \pi^{2}$ for the other equilibrium points. $\lambda_{3}$ is always positive since $p_{3}$ is a positive real parameter. So, these equilibria $(P(0,0, k \pi), k \in \mathbb{Z}$ and $k \neq 0)$ are nonhyperbolic and characterized by a $2 \mathrm{D}$ center manifold and $1 \mathrm{D}$ stable manifold. The numerical investigations show that all equilibrium points are unstable. An equilibrium point is stable when trajectories starting in its proximity remain close or converge toward it.

\section{Numerical Analysis of the New System}

In this section, we will show the dynamical behaviors of system (1). The use of well-known tools such as bifurcation diagram, Lyapunov Exponents (LEs) spectrum, phase portrait, and SE complexity helps us to demonstrate the chaotic behavior of the proposed system. The largest LE is an important tool for detecting chaos. SE algorithm is a powerful measure of the chaotic characteristics of the system Liu et al. [54]. Here, all the simulations are made by using numerical tools based on an ode45 solver with a time step fixed at $\Delta t=0.001 \mathrm{~s}$.

3.1. Bifurcation Behavior. In this subsection, the bifurcation diagrams and the corresponding LE spectrums with respect to the control parameters such as $p_{1}, p_{5}, p_{6}$, and $p_{7}$ have 
been investigated in detail. At first, to reveal the dynamic behaviors of system (1), we consider the parameter $p_{1} \in[3.92,4.1]$. For the parameters $\left(p_{2}, p_{3}, p_{4}\right.$, $\left.p_{5}, p_{6}, p_{7}\right)=(7,7,5,4.5,2.9,1)$ and initial value $(-1,-1,-1)$, the bifurcation diagram and the corresponding LE spectrum with respect to $p_{1}$ are shown in Figure 2 . When the system parameter $p_{1}$ is increased from 3.92 to 4.1, Figure 2(a) shows that the system changes from chaotic state to periodic state, alternatively. There exists a periodic window in $p_{1}=3.929$, and we observe a periodic motion with order-5 when $p_{1}=3.93$. Also, chaotic bubbles are presented in Figure 2(a) (for $p_{1} \in[4.03,4.1]$ ) that exhibit a complex behavior of the nonlinear system. In addition, the spectrum of LEs in Figure 2(b) well matches the bifurcation diagram. As it is known, when $\mathrm{LE}_{1}>0, \mathrm{LE}_{2}=0$, and $\mathrm{LE}_{3}<0$, the proposed system is in a chaotic state.

When $p_{5}$ varies from 4.2 to 5.3 , the bifurcation diagram and corresponding Ls spectrum with initial values $(-1,-1,-1)$ are plotted in Figures 3(a) and 3(c), respectively. Figure $3(\mathrm{a})$ shows that the system is alternately changing from a nonchaotic state to a chaotic state. For some values of $p_{5}$, the system has a small positive maximum LE, and the system is in a weak chaotic state. As shown in Figure 3(c), there are large fluctuations in the curve, which indicates the change has a great impact on the dynamical behavior of system (1).

Also, the bifurcation diagram and LEs spectrum versus the parameter $p_{6} \in[3.94,4.05]$ are presented in Figures 3(b) and 3(d). The bifurcation diagram in Figure 3(b) shows that the local maxima of the coordinate $z$ directly illustrate an important phenomenon. This phenomenon is period-adding bifurcation, and we observe two sequences that overlap with each other. In this phenomenon, the oscillation period successively increases in different sequences by system parameter alteration.

Let the system parameters be $\left(p_{1}, p_{2}, p_{3}, p_{4}, p_{5}\right.$, $\left.p_{6}\right)=(4.4,7,7,5,4.5,2.9)$ and initial value $(-1,-1,-1)$. The bifurcation diagram versus $p_{7} \in[0.25,1.3]$ is plotted in Figure 4(a). As it can be seen from this diagram, there are disconnected chaotic and periodic areas. For more details and revealing more interesting phenomena, the red frames of the bifurcation diagram of Figure 4(a) are magnified in Figure 5 and Figures 4(b) and 4(c), respectively. In the next subsection, we address the period- 2 bubbles that are observed on the left side of the graph in Figure 4. Chaotic motions, periodic windows, and period-doubling bifurcation are also seen in Figure 4(b). The intermittent transition from period-1 motion to chaotic motion occurred in $p_{7}=$ 0.9881 (In Figure 4(c)). Also, the period-3 window occurs near $p_{7}=1.02$ after reverse period-doubling. Moreover, the route to chaos via period-doubling bifurcation is strongly visible in Figure 4(c).

3.2. Antimonotonicity. The phenomenon of antimonotonicity has been found in many dynamical systems including 3D autonomous systems without linear terms in Kengne et al. [30]. In this phenomenon, periodic orbits can be created and then destroyed via reverse period-doubling bifurcation as a control parameter is varied (ZolfaghariNejad et al. [16]). In order to demonstrate this phenomenon in system (1), we have illustrated some bifurcation diagrams as the parameter $p_{7}$ is varied in the range $0.52<p_{7}<0.75$ for some discrete values of the control parameter $p_{6}$. Sample results are shown in Figure 5. In Figure 5(a), four period-2 bubbles are observed in $p_{6}=2.9$ (the left red frame in Figure 4(a)). Also, we have stable period-4 bubbles at $p_{6}=2.97$ (Figure 5(b)). Similarly, more bubbles are created for $p_{6}=3.01$ (Figure 5(c)), and an infinite tree finally occurs at $p_{6}=3.15$ (full Feigenbaum remerging tree in Figure 5(d)).

3.3. Multistability. In order to uncover the coexisting characteristic, we select the system parameters as $\left(p_{1}, p_{2}, p_{3}\right.$, $\left.p_{4}, p_{5}, p_{6}, p_{7}\right)=(4,7,7,5,4.785,4,1)$, and let the initial value be $\left(-1,-1, z_{0}\right)$. Figure 6 presents the phase portraits of eight coexisting attractors of system (1). However, they are samples of an infinite number of attractors which form along the $z$-axis in phase space and could be detected with other initial values.

The coexistence of multiple periodic, limit cycle, and chaotic attractors with different topologies is one of the most attractive properties considered in this work. The corresponding bifurcation diagram versus $z_{0}$ as a control parameter is provided in Figure 6(a), which can be observed as multistability in system (1). When $z_{0}=-1$, we can observe a strange attractor in Figure 6(c). For the initial value $z_{0}=-1.1$, the existence of the order-3 period is proven in Figure 6(d). In Figures 6(e) and 6(f), order-1 period, limit cycle, and chaotic attractors coexist together for selected initial values, and they are asymmetric.

3.4. Spectral Entropy Analysis. Spectral entropy has been used to determine the unpredictability of time-series Kengne et al. [30]. Spectral entropy is defined as

$$
\mathrm{SE}(N)=-\sum_{k=1}^{N} P_{k} \ln P_{k},
$$

where $P_{k}$ is given by

$$
P_{k}=\frac{\left|\lambda_{k}\right|^{2}}{\sum_{i}\left|\lambda_{i}\right|^{2}}
$$

Here, $\left|\lambda_{k}\right|^{2}$ is the Fourier power spectrum of a time-series at frequency $\lambda_{k}$ Legendre and Legendre [55].

For the purpose of revealing the effect of the two parameters $p_{1}$ and $p_{6}$ on the chaotic behavior of system (1), we plot the spectrum of SE complexity as Figure 7(a). Fixing $\left(p_{2}, p_{3}, p_{4}, p_{5}, p_{7}\right)=(7,7,5,5.005,1)$, the initial value $(-1$, $-1,-1)$, and varying $p_{1}$ and $p_{6}$ from 3 to 5 , SE distribution of the system is obtained as Figure $7(\mathrm{a})$. The darker areas are separated by a curved line. Also, a curved line is observed in lighter areas where around it the more chaotic state and nonchaotic state are repeated alternatively. We use the SE complexity algorithm in parameter selection for the novel system in real applications. 


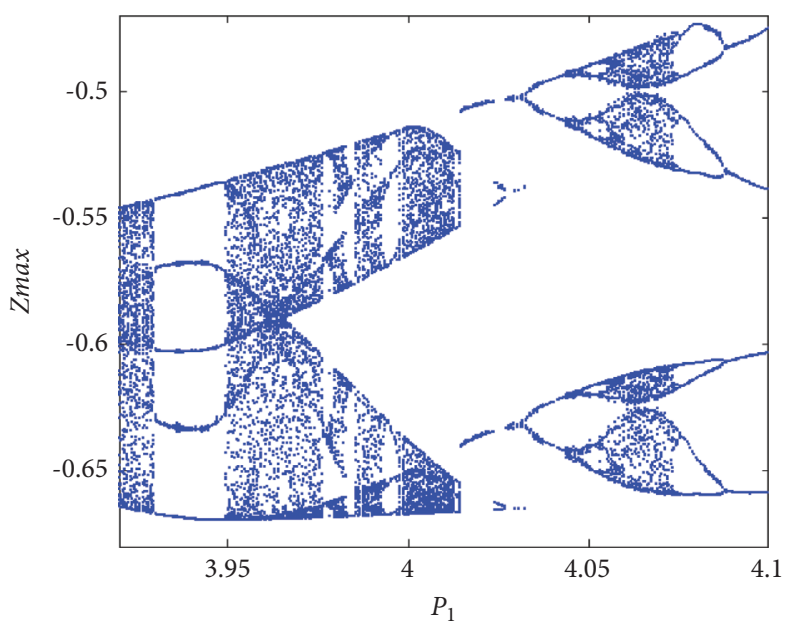

(a)

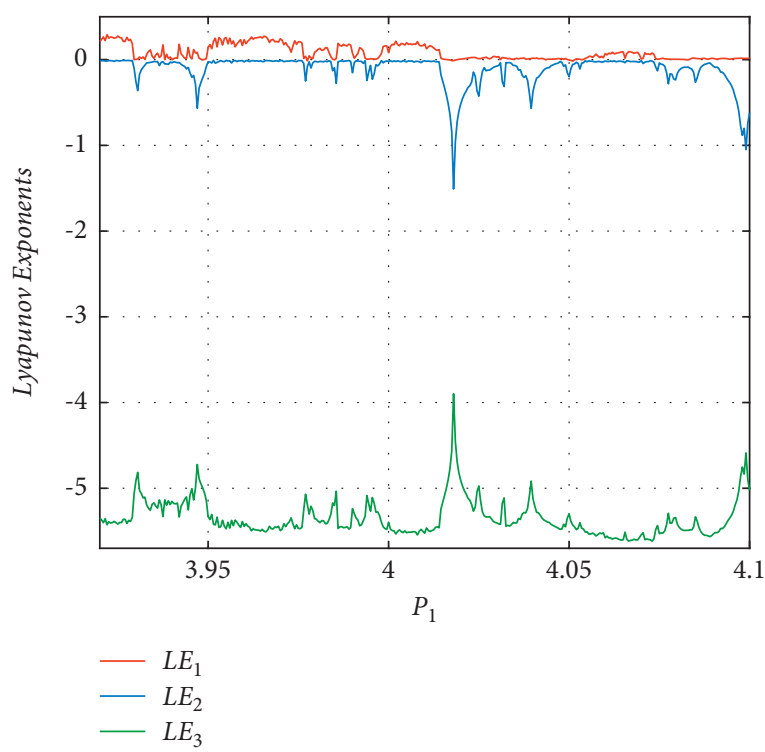

(b)

Figure 2: Bifurcation diagram and LEs spectrum of system (1) with the initial value $(-1,-1,-1)$ and $\left(p_{1}, p_{2}, p_{3}, p_{4}, p_{5}, p_{6}, p_{7}\right)=\left(p_{1} \in[3.92,4.1], 7,7,5,4.5,2.9,1\right)$. (a) Bifurcation diagram; (b) LEs spectrum.

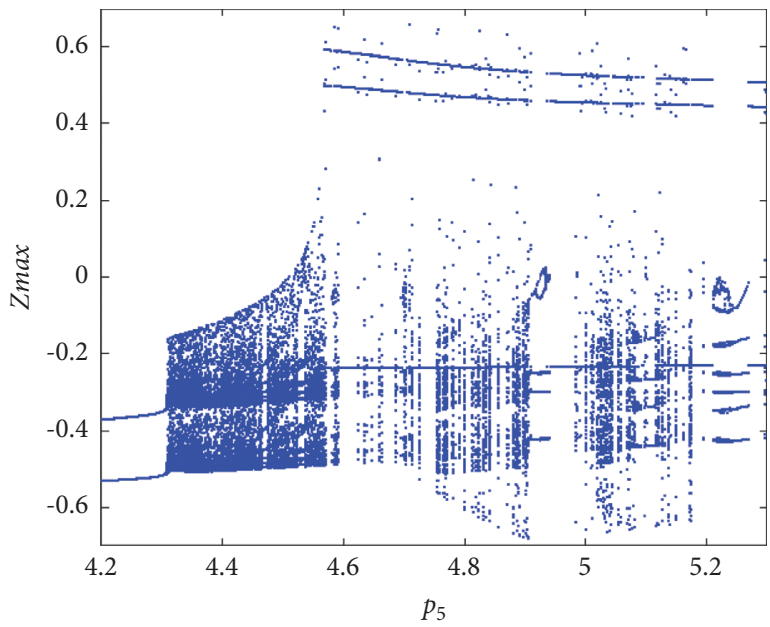

(a)

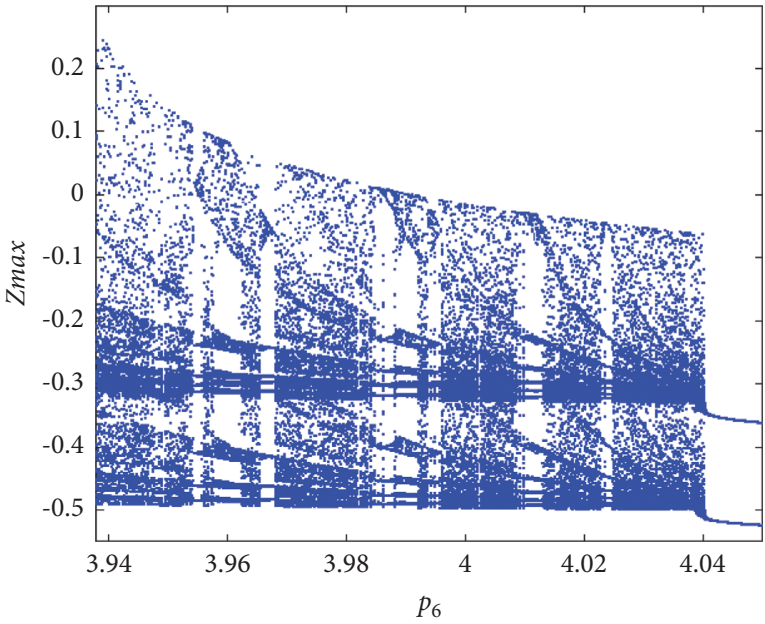

(b)

FIGURE 3: Continued. 


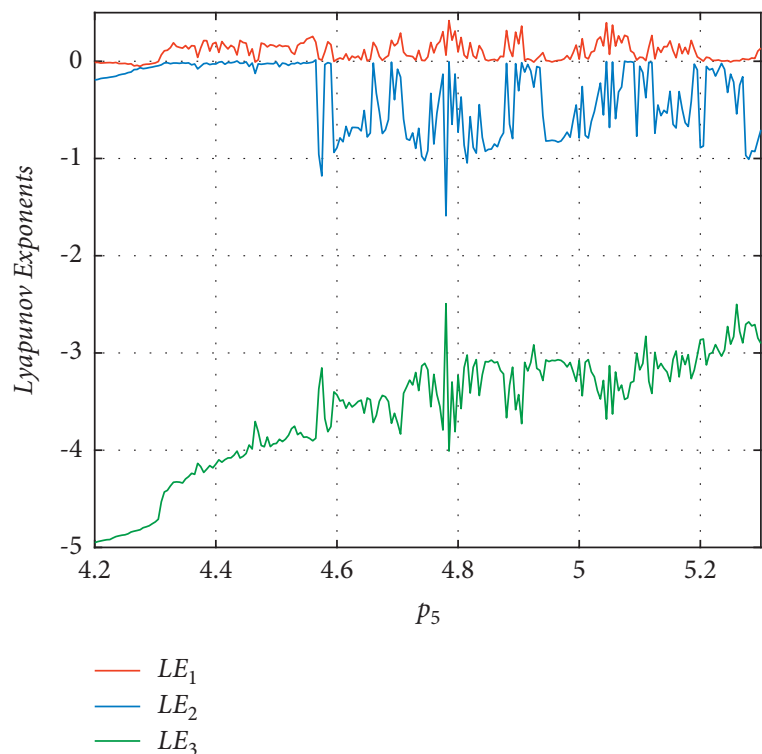

(c)

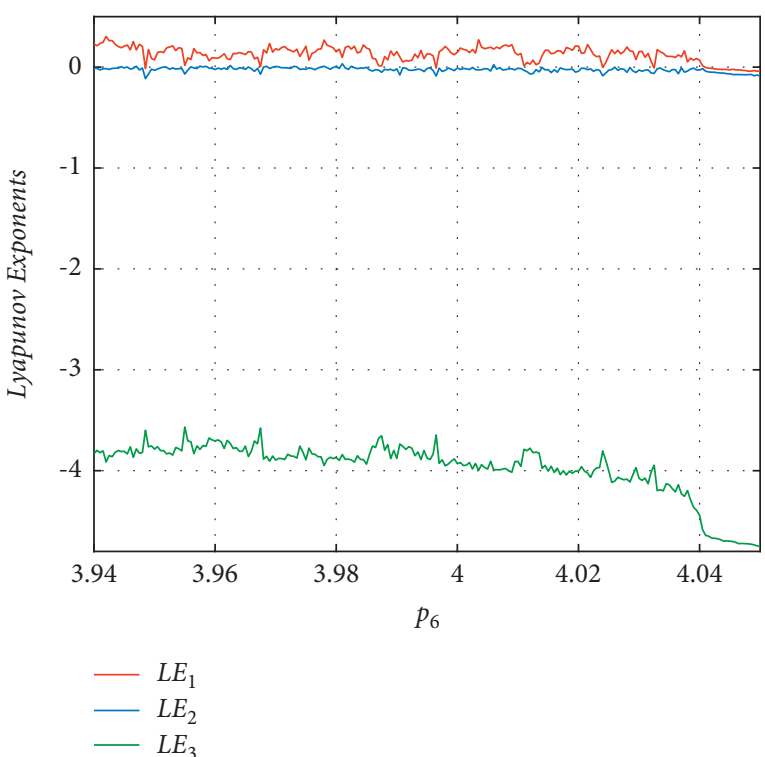

(d)

Figure 3: Bifurcation diagrams and LEs spectrum of system (1) with the initial value $(-1,-1,-1)$ and $\left(p_{1}, p_{2}, p_{3}, p_{4}, p_{5}, p_{6}, p_{7}\right)=(4,7,7,5,1)$. (a) Bifurcation diagram with respect to $p_{5}\left(p 5 \in[4.2,5.3], p_{6}=2.9\right)$. (b)Bifurcation diagram with respect to $p_{6}\left(p_{5}=4.5, p_{6} \in[2.94,4.05]\right)$. (c) The corresponding LE spectrum with (a). (d) The corresponding LE spectrum with (b).

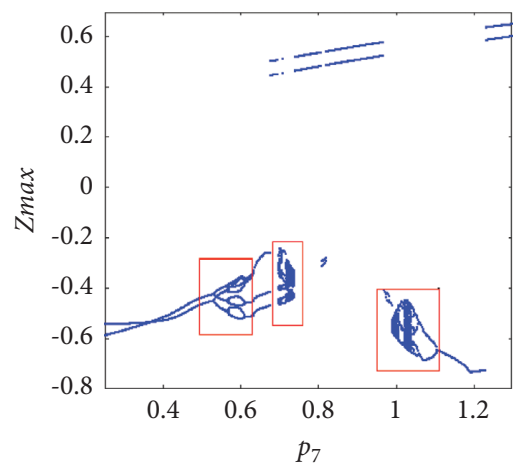

(a)

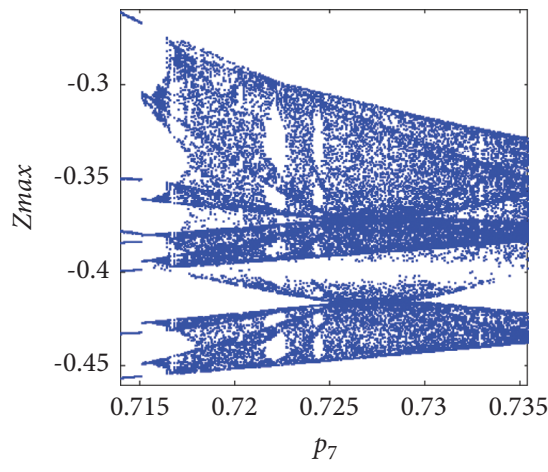

(b)

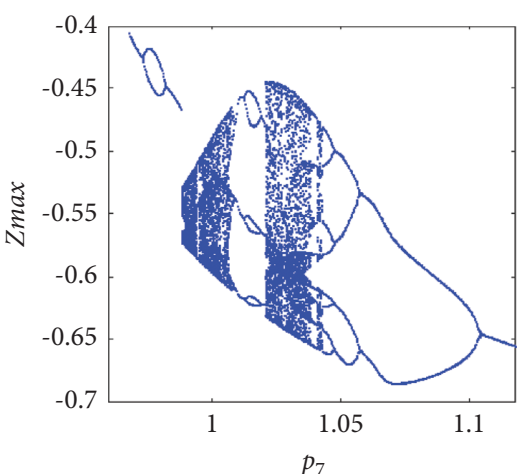

(c)

Figure 4: (a) Bifurcation diagram of the system with $\left(p_{1}, p_{2}, p_{3}, p_{4}, p_{5}, p_{6}, p_{7}\right)=\left(4.4,7,7,5,4.5,2.9, p_{7} \in[0.25,1.3]\right)$ and initial value $(-1$, $-1,-1)$. A magnified version of the bifurcation diagram versus (b) $p_{7} \in[0.714,0.735]$ and (c) $p_{7} \in[0.968,1.118]$.

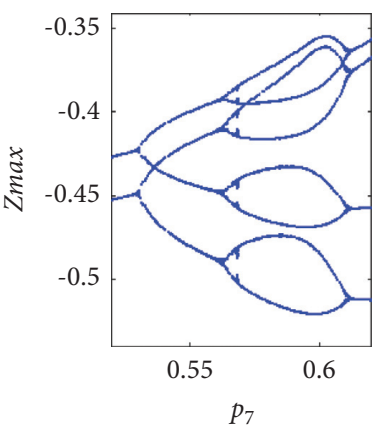

(a)

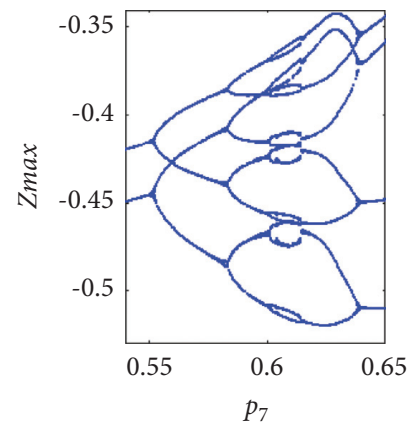

(b)

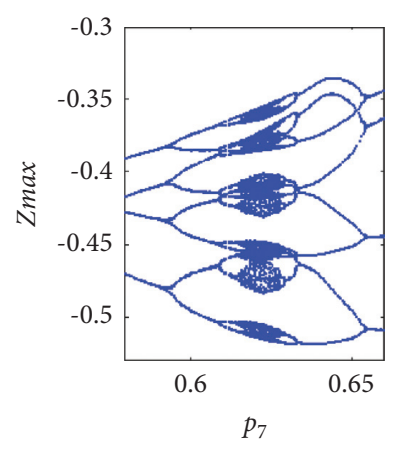

(c)

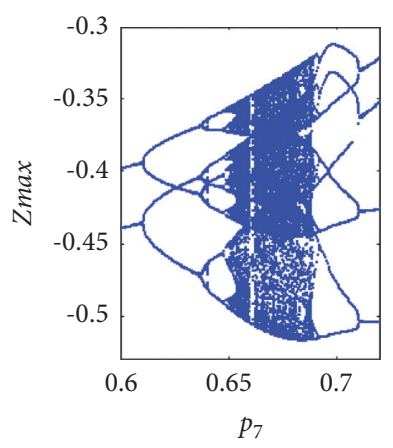

(d)

FIgURE 5: Bifurcation diagram for remerging Feigenbaum tree (bubbling): (a) period-2 bubble for $p_{6}=2.9$; (b) period- 4 bubble for $p_{6}=2.97$; (c) and (d) full Feigenbaum remerging tree at $p_{6}=3.01$ and $p_{6}=3.15$; the initial value is $(-1,-1,-1)$. 


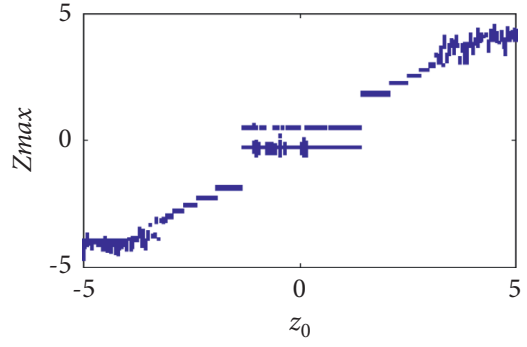

(a)

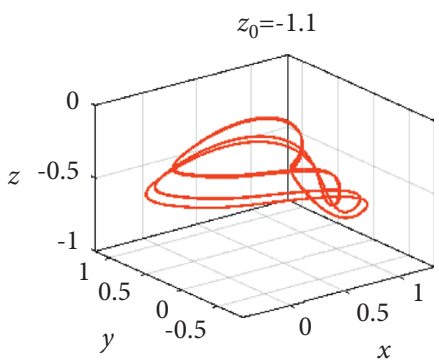

(d)

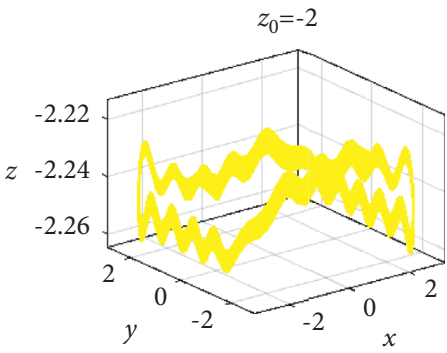

(g)

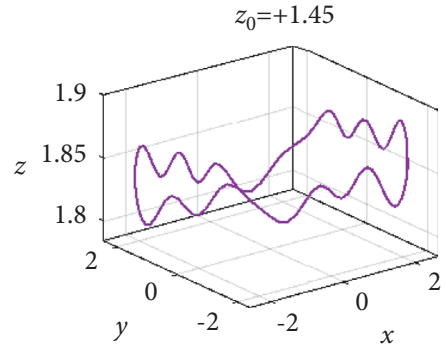

(b)

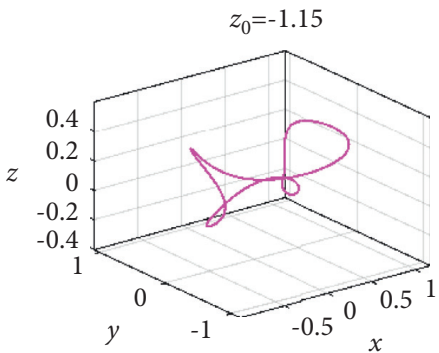

(e)

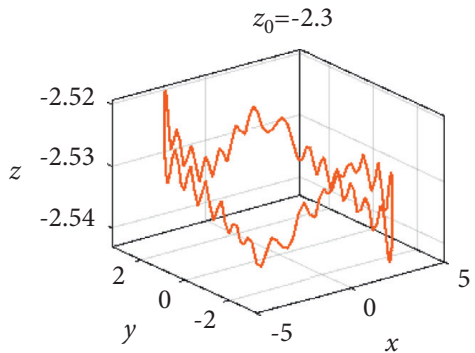

(h)

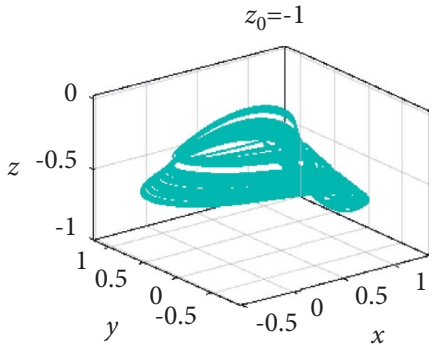

(c)

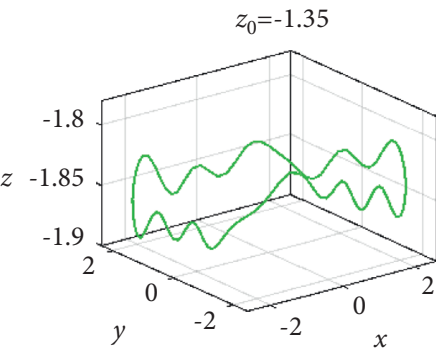

(f)

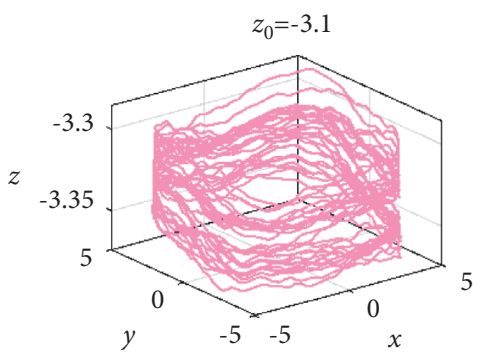

(i)

Figure 6: Sample phase portraits of system (1) under $\left(p_{1}, p_{2}, p_{3}, p_{4}, p_{5}, p_{6}, p_{7}\right)=(4,7,7,5,4.785,4,1), x_{0}=-1, y_{0}=-1$ showing the occurrence of multiple attractors. (a) Bifurcation diagram of the system versus $z_{0}$, (b) period-1 cycle, (c) spiral chaotic attractor, (d) period-3 cycle, (e, f) period-1 cycle with different topologies, (g) limit cycle, (h) period-1 cycle, and (i) limit cycle.

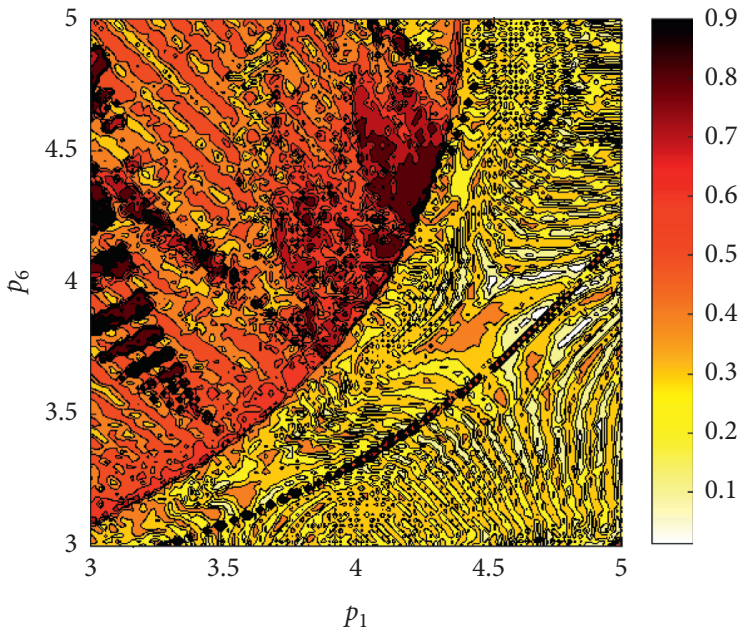

(a)

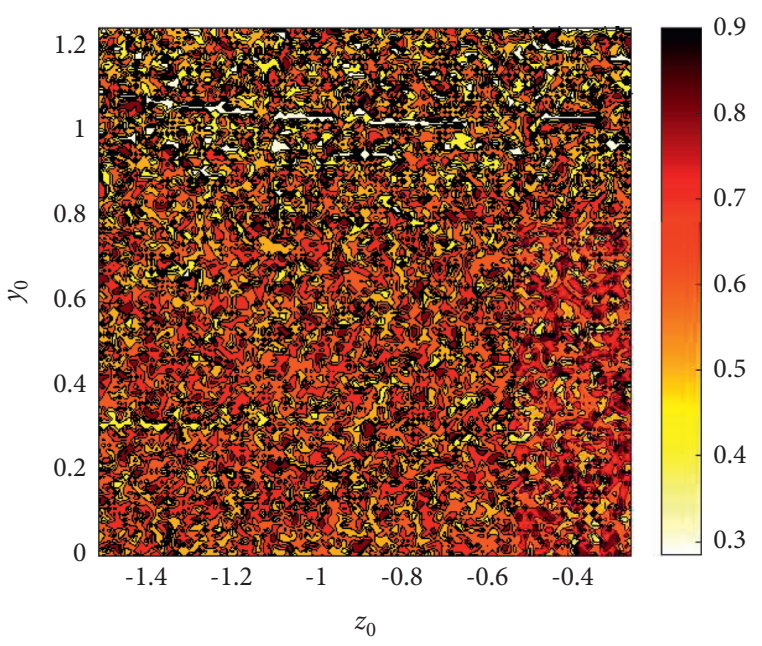

(b)

Figure 7: Spectral entropy distribution of the system in the (a) $p_{6}-p_{1}$ plane and (b) $y_{0}-z_{0}$ plane. 
We can see the SE distribution of system (1) in $y_{0}-z_{0}$ plane in Figure $7(\mathrm{~b})$. Here, the system parameters remain unchanged and $p_{1}=4, p_{6}=4$, and $x_{0}=-1$. The SE complexity is computed when $z_{0} \in[-1.5,-0.25]$ and $y_{0} \in[0,1.25]$. Figure $7(\mathrm{~b})$ reflects the SE distribution under different initial values of $y_{0}$ and $z_{0}$. The color distribution in $y_{0}-z_{0}$ plane is very scattered which demonstrates that the novel system is very sensitive to the initial values. Other than small areas with lighter colors at the top of the plane, in the entire area, numerous small black spots are diffused. Moreover, this figure illustrates that different initial values have a great impact on the complexity of system (1). Also, we observe that the system has multiple coexisting attractors.

\section{A Secure Data Transmission Scheme Using the Proposed Chaotic System}

In this section, we give a complete description of the proposed secure data transmission scheme. Chaotic systems are used as one of the potential alternative encoding techniques in secure data transmissions. Chaos-based encoding/ decoding algorithms offer high flexibility in secure data transmission schemes due to numerous numbers of chaotic systems and many possible secret keys. However, chaotic systems are often applied in designing pseudorandom number generators (PRNGs) with a high degree of randomness as the most basic unit in secure transmission schemes. In this work, the proposed chaotic system has been used to design a new encoding/decoding algorithm with an evolutionary approach.

Deterministic secure data transmission schemes have predictable behavior because there is always only one output for each constant data. So, these schemes can never amount to the level of high security. In contrast, a probabilistic scheme produces different outputs at different times even when the algorithm is applied with the same key to the same input data. As a result, probabilistic secure transmission schemes possess resistance against chosen-plaintext and ciphertext-only attacks (Dhall et al. [45]). In this section, we propose a new inherently probabilistic scheme that provides high security and has unpredictable behavior.

4.1. Problem Formulation. Figure 8 summarizes the secure data transmission setup. Suppose Alice wants to send an $l$-bit message $m=\left(m_{i}\right)_{i=1}^{l} \in\{0,1\}^{l}$ through the public channel to Bob. She first encodes the message by a chaos-based encoding algorithm and produces a set of codewords. While encoding a message, she utilizes the preagreed secret key which may drive the encoding step when producing codewords. Codewords $c=\left\{c_{0}, \ldots, c_{s}\right\}$ are sent through the channel and inspected by Eve. Suppose Eve wants to understand the confidential message between Alice and Bob; however, she does not have the secret key. If codewords are delivered to Bob, he extracted the message by inverting the encoding operation via a chaos-based decoding algorithm.

In this work, chaos-based encoding/decoding algorithms are realized by applying a designed SC diagram using different steady states. There are several possible final steady states for a set of parameters in chaotic systems with multiple coexisting attractors. The final state depends on the initial values of the system and is determined by an evolutionary rule that the system goes from the current state to which state. In these systems, the SE distribution of the system under different initial values is very dispersive (see Figure 7(b)). Our main idea for encoding a message is also the use of SE complexity distribution.

The SC diagram determines the relationship between the steady states of the system and message bits. Figure 9 shows a simple designed SC diagram. In this structure, three final states $A, B$, and $C$ are considered. Each edge is labeled with $q_{i} \in\{0,1\}$, where it shows one bit of message $m$. For each bit of the message, the sender generates the corresponding codeword, so that the chaotic system evolves into a final steady state in the receiver. Also, it should be noted that when assigning $q_{i}$, to (from) each state, at least one " 0 " and one " 1 " should be entered (be exited). The assignment process of $q_{i}$ to edges is pseudorandom and can be considered as a part of the secret key. We have different paths through the SC diagram that lead all paths toward a specified message. This is due to the existence of several possible final steady states and many different initial values for the evolution of the system. The number of paths varies depending on the length of the message, the number of considered states, and the number of initial values for the realization of each state.

The final steady states $A, B$, and $C$ can be obtained under different initial values. For instance, Figure 10 indicates three different states obtained by $y_{0} \in[-1.5,-0.25]$ and $z_{0} \in[0,1.25]$, which reflects the SE distribution of system (1). The system parameters and $x_{0}$ are fixed as shown in Section 3.4. Figure 10(a) shows the dispersion of $\mathrm{SE} \leq 0.55$ with black dots (state $A$ ). The state $B$ is defined as $0.55<\mathrm{SE}<0.7$ (Figure 10(b)), and state $C$ is obtained by $\mathrm{SE} \geq 0.7$ as shown in Figure 10(c). As is shown in these figures, there are many initial values for the realization of each state. Also, the distribution of these initial values in each state is random and unpredictable.

4.2. Algorithm Description. The proposed algorithms for the encoding and decoding procedure are described in Figure 11 using pseudocode and based on the SC diagram of Figure 9. For the decoding procedure, we need to know how to label the edges in the SC diagram $\left(q_{i}\right)$. Therefore, we are able to reconstruct the paths of the SC diagram in the receiver. As mentioned, the process of assigning the labels is considered as a part of the secret key shared between the sender and the receiver.

In this construction, the length of codewords can be $n \in\{4,8,16, \ldots\}$ according to the SC diagram. The codewords refer to the initial value that creates a determined state. The set of codewords $c=\left\{c_{0}, \ldots, c_{s}\right\}$ generated for each message can be represented as a path through the SC diagram. So, different sets of codewords can be sent each time for a fixed message. In this section, we first describe the encoding/decoding algorithms in their general form, and later, in Section 4.2.1, we give two simple examples for better understanding. 


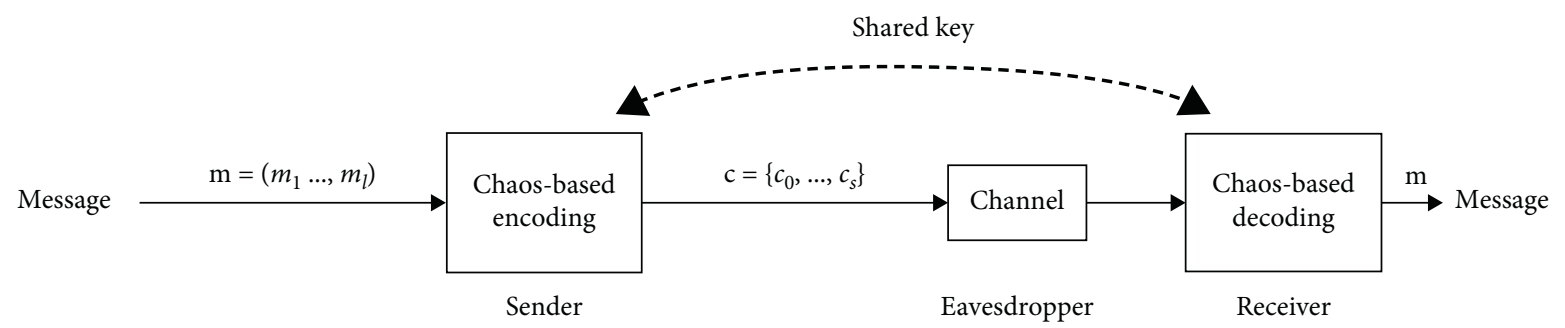

Figure 8: Model of secure data transmission scheme.

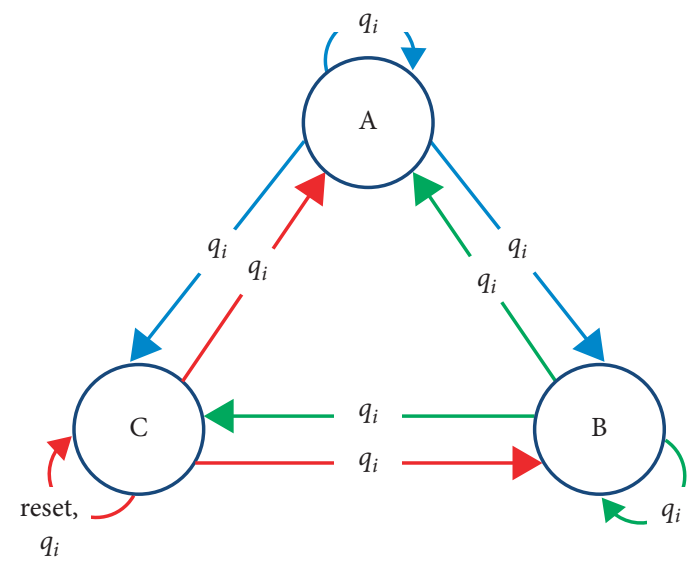

Figure 9: The state chain diagram with $q_{i} \in\{0,1\}$.

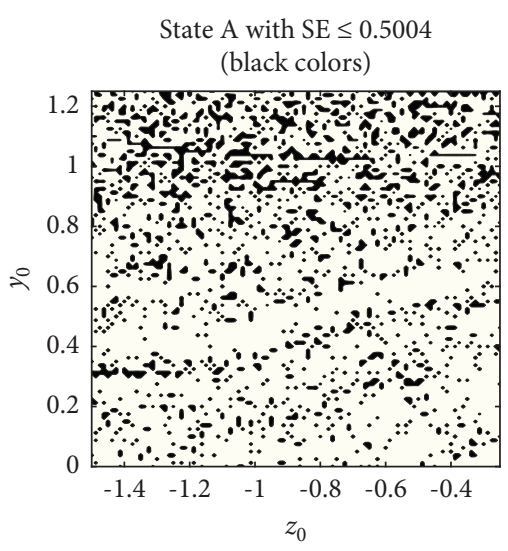

(a)

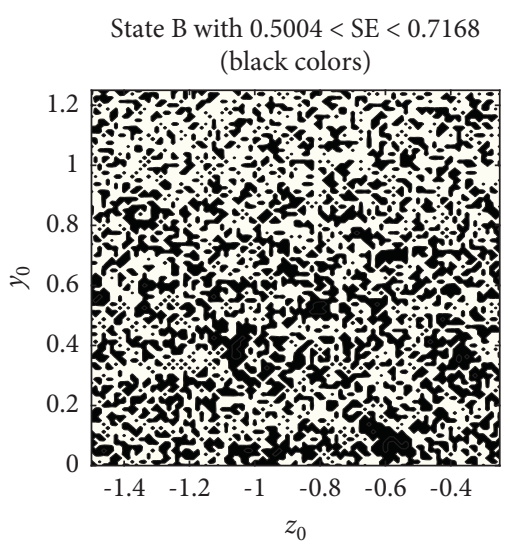

(b)

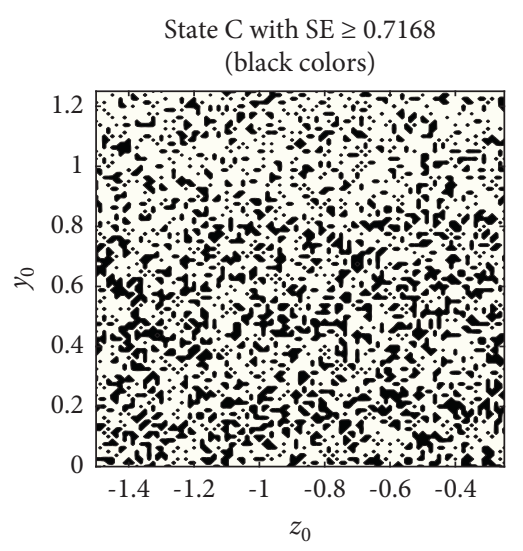

(c)

FIgURE 10: Dispersion of spectral entropy in the $y_{0}-z_{0}$ plane, (a) $\mathrm{SE} \leq 0.55$, (b) $0.55<\mathrm{SE}<0.7$, and (c) $\mathrm{SE} \geq 0.7$.

In Algorithm 1 of Figure 11 (Encoding procedure), the SE complexity of the chaotic system (1) under different initial values is first computed, and then one codeword will be assigned to each initial value. Also, the states $A, B$, and $C$ are set based on computed spectral entropy. In general, the message $m$ is divided into equal blocks $b=\left\{b_{1}, \ldots, b_{s}\right\}$. In fact, the encoding process is done in block form. Based on the SC diagram in Figure 9, there are three steady states. So, the length of each block is equal to one, and each block represents one bit of the message $(l=s)$.
In the following, one state is randomly selected named as the current state. Depending on the current state, one codeword is randomly selected $\left(c_{0}\right)$. As we explain this procedure, the reader is advised to inspect Algorithm 1 of Figure 11. According to the SC diagram, the current state, and the first block of $m\left(b_{1}\right)$, the next state is determined. The edge between the current state and the next state is labeled with $q_{i}=b_{j}$. Then, another codeword is randomly selected according to the next state $\left(c_{j}\right)$. In the following, the next state replaces the current state, and the process is repeated. 


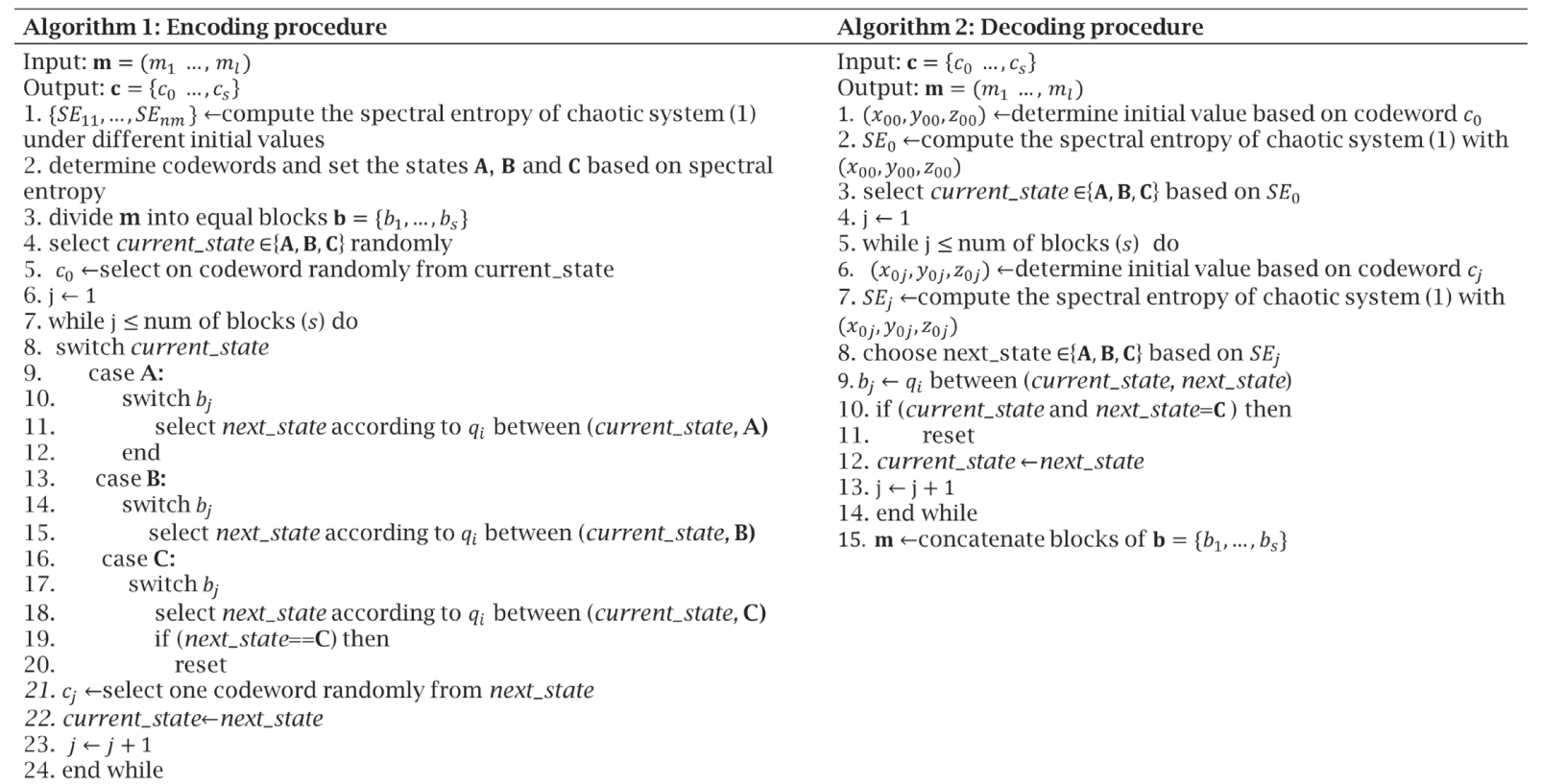

Figure 11: Pseudocode of the encoding and decoding procedures.

This algorithm continues until all message blocks are mapped to the corresponding codewords.

In the encoding procedure, whenever the state $C$ is repeated twice times in succession, the algorithm resets. Therefore, depending on the current block of $m$, the system parameters are changed. Then, the SE complexity of system (1) under initial values is recomputed. This process causes a more complicated algorithm. As a result, the proposed algorithm becomes more resistant to eavesdropping attacks. Finally, a generated set of codewords $c=\left\{c_{0}, \ldots, c_{s}\right\}$ is sent to the receiver.

The decoding procedure (Algorithm 2 of Figure 11) starts with the first transmitted codeword $\left(c_{0}\right)$, and the initial value $\left(x_{00}, y_{00}, z_{00}\right)$ is determined based on it. Then, the SE complexity of system (1) is calculated by knowing the initial value and system parameters extracted from the secret key. $\mathrm{SE}_{0}$ determines the current state. In the same way, the next codeword specifies the next state. In the SC diagram, the edge that connects the current state with the next state is labeled $q_{i}$ and assigns the first block of the message $m\left(b_{1}\right)$. The next state replaces the current state, and the second codeword $\left(c_{1}\right)$ determines the initial value $\left(x_{01}, y_{01}, z_{01}\right)$. By calculating the SE complexity of the system, the next state is specified, and the second block of $m\left(b_{2}\right)$ is obtained. The decoding procedure continues until all blocks of $m$ are specified. Finally, the blocks $b=\left\{b_{1}, \ldots, b_{s}\right\}$ concatenates, and the confidential message is restored.

4.2.1. Two Examples of the Proposed Algorithm. In this subsection, the algorithm for secure data transmission is explained with two small examples. Let the labels assignment of the edges in the SC diagram of Figure 9 be as in Figure 12(a). We assume $l / s=1$ and thus $q_{i}$ represent one bit of message $m$. In the first example, suppose that the message is $m_{1}=(0,1,1,0)$. As shown in Figure $12(\mathrm{~b})$, the encoding algorithm starts with a random selection of one of the states $(A, B, C)$. Suppose state $B$ is selected. Depending on state $B$, one codeword is randomly selected $\left(c_{10}\right)$. This codeword refers to the initial value which causes system (1) to evolve to the state $B$. Two edges are exited from this state that is labeled " 0 " $\left(m_{11}\right)$. The first edge connects state $B$ to state $C$, and the second edge connects $B$ to itself. We select one of the states $C$ or $B$, randomly. Suppose that state $B$ is selected again as the next state. So, the second codeword randomly picks up from state $B\left(c_{11}\right)$. Depending on the second bit of $m_{1}$, one edge is exited from this state that is labeled " 1 ". Then, the next state is $A$, and $c_{12}$ is determined. The state $A$ has two exited edges labeled " 1 " that equals to $m_{13}$. These edges connect state $A$ to the states $B$ and $C$. With random selection between $B$ and $C, c_{13}$ is also determined. If the state $C$ is selected, the final state will be $A$, because just one exited edge from $C$ is labeled " 0 " (according to the last bit of $m_{1}$ ). So, $c_{14}$ is randomly selected from state $A$. The codewords $c=\left\{c_{10}, c_{11}, c_{12}, c_{13}, c_{14}\right\}$ are sent through the public channel and are delivered in the receiver.

In the receiver, $m_{1}$ is restored using the secret key by running Algorithm 2 of Figure 11. In the first step, $\left(x_{00}, y_{00}, z_{00}\right)$ is determined based on the codeword $c_{10}$, and then $\mathrm{SE}_{0}$ is calculated for this initial value. Also, based on $\mathrm{SE}_{0}$, the current state is determined. The second initial value $\left(x_{01}, y_{01}, z_{01}\right)$ is determined by the codeword $c_{11}$, and the corresponding $\mathrm{SE}$ of system (1) is computed. Indeed, $\mathrm{SE}_{1}$ specifies the next state. The edge between the current state and the next state labeled $q_{i}$ corresponds to the first bit of the message $m_{1}$. So, the next state replaces the current state, and the third initial value resulting from $c_{12}$ determines the next state. This procedure continues until the last bit of $m_{1}$ has been extracted. 


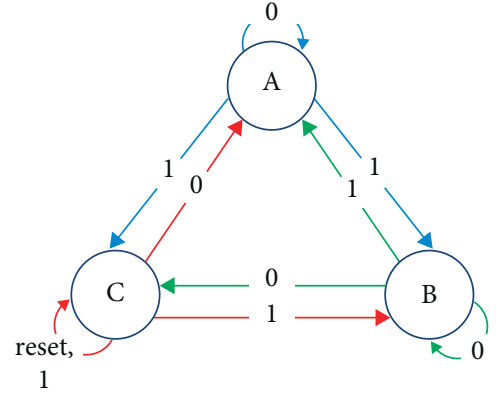

(a)

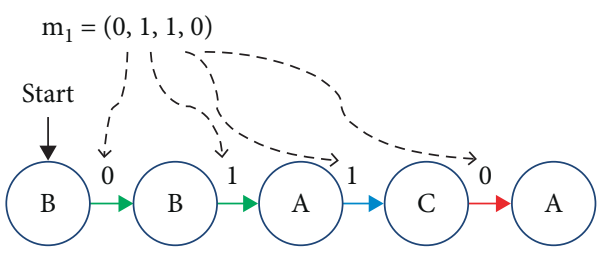

(b)

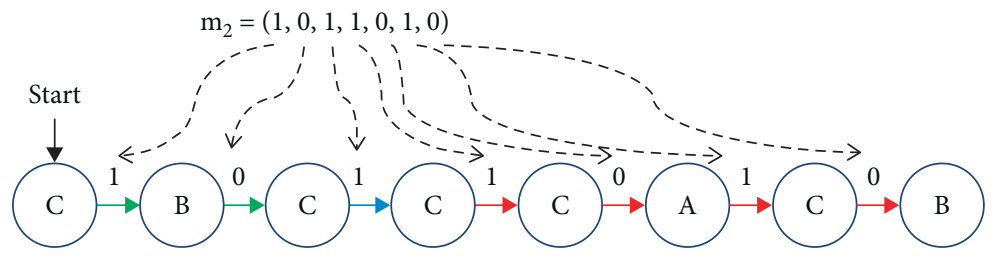

(c)

FIgURE 12: (a) The state chain diagram with assignments. (b) Selected path through the SC diagram of (a) for $m_{1}=(0,1,1,0)$. (c) Selected path through the SC diagram of (a) for $m_{2}=(1,0,1,1,0,1,0)$.

In the second example, suppose the message is $m_{2}=(1,0,1,1,0,1,0)$. Figure $12(\mathrm{c})$ represents the selected path through the SC diagram of Figure 12(a) for this example. Suppose state $C$ is randomly selected first as the current state. Depending on state $C$, one codeword is randomly selected. Two edges that connect state $C$ with itself and state $B$ are labeled " 1 " (according to $m_{21}$ ). Suppose state $B$ is selected as the next state. So, the next codeword is randomly determined based on state $B$. In this step, state $B$ replaces the current state, and the second bit $m_{2}\left(m_{22}\right)$ determines the next state. Then, the next state is $B$ or $C$. Suppose the state $C$ is selected, and the third codeword is randomly chosen from state $C$. Similarly, $m_{23}$ determines the next state. Suppose the next state is state $C$, again. Therefore, state $C$ is repeated twice times in succession, and the encoding algorithm resets. Depending on the current bit of $m_{2}$, we change the system parameters based on the preshared agreement between the sender and the receiver. So, we recompute SE complexity of system (1) under initial values. Then, the codeword $c_{3}$ is randomly chosen from new state $C$. This algorithm continues to encode the other message bits. At the receiver, depending on the received codewords, the initial values are extracted, and the system evolves to the desired states based on the SC diagram. As a result, we can extract the message $m_{2}$ correctly on the receiver side.

4.2.2. Extended State Chain Diagram. The SC diagram in Figure 9 is an example of a simple model, in which each state represents the final steady state of the chaotic system (1) based on the SE complexity. In this model, the length of each block of $m$ is one, and each edge is labeled with $q_{i}$ equal to one bit of the message. As a result, the number of transmitted codewords is equal to the number of message bits. When the message length is short, the number of generated codewords is low enough. However, by increasing the message length, the number of codewords is increased. We can design an extended SC diagram with more states to improve the efficiency of the proposed secure transmission scheme. For example, the extended SC diagram in Figure 13 has nine states. These states can be obtained by dividing the SE distribution of Figure 7(b) into nine parts. Each edge is labeled with $r_{i} \in\{000,001,010,011,100,101,110,111\}$ which represents different modes for each block of $m$. Therefore, one codeword is generated for one block of the message with a length of three bits. As a result, the number of generated codewords is decreased than before.

\section{Performance Analysis and Experimental Results}

In this section, experimental observations are given to demonstrate the performance and validity of the proposed scheme. In Section 4, we described the details of the proposed chaos-based encoding/decoding algorithms for a secure data transmission scheme. As mentioned, the transferred data through the public channel is a set of codewords in the form of $c \in\{0,1\}^{n}$. Each codeword refers to the initial value which causes system (1) to evolve to the desired state. At the receiver, with the dynamic evolution of the chaotic system, the original data is restored based on the SC diagram. The proposed scheme is independent of data type, and we can send any type of confidential digital data such as image, text, and numeric data. Also, this scheme is capable of sending data in different paths through the SC diagram. As a result, the proposed scheme is inherently probabilistic and provides high security. For the evaluation of the proposed scheme, we used the extended SC diagram in Figure 13. 


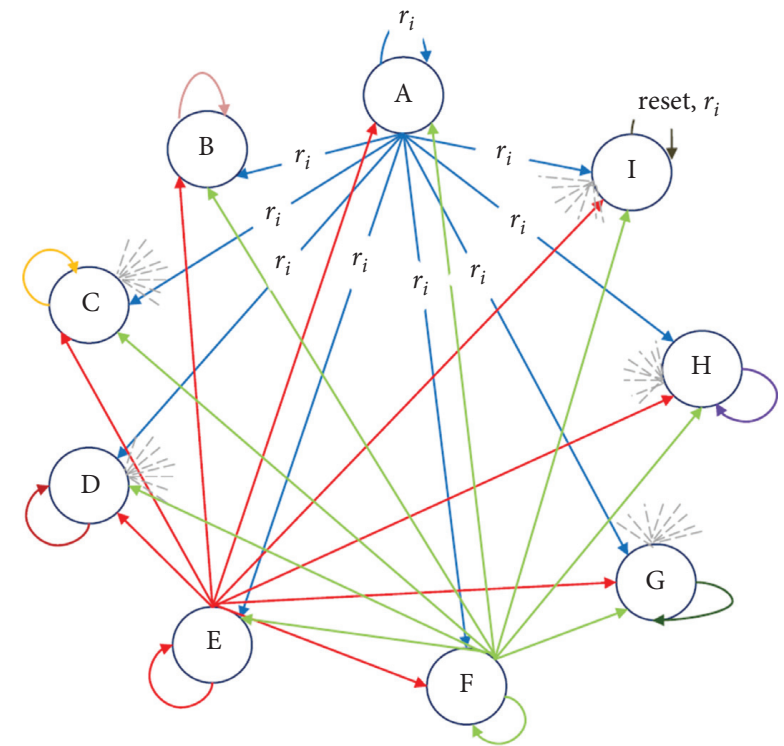

FIGURE 13: Extended state chain diagram $\left(r_{i} \in\{000,001, \ldots, 111\}\right)$.

5.1. Security Analysis. At first, common security analyses are evaluated including keyspace and sensitivity to mismatched keys. Then, statistical analyses are examined.

(1) Keyspace. For the evolution of the dynamical system, we need to know the parameters of the system. Also, how to label the edges with $q_{i}$ (or $r_{i}$ ) in the SC diagram is required. The proposed scheme uses 17 parameters as keys, and these keys are coded with $17 \times 16$ bits. Then. the total keyspace is equal to $2^{272}$. The keyspace of this framework is greater than $2^{100}$ and is considered secure (Alvarez and $\mathrm{Li}$ [56]). So, the proposed scheme is infallible against brute force attacks. (2) Sensitivity to Mismatched Secret Key. We tested the sensitivity of the proposed scheme to the mismatched secret key. The dynamical system is very sensitive to small changes in the value of the parameters. Thus, even one wrong bit in the secret key results in a completely different message at the receiver. Figure 14 shows the original image and the extracted image at the receiver with one wrong bit in the secret key. In this case, one bit of the secret key is randomly selected and is flipped. (3) Dissimilarity Matrix for a Constant Message. The proposed chaos-based encoding algorithm is capable of sending data in different paths. Since there are many initial values for each state, the proposed algorithm corresponding to the SC diagram has different paths for data transmission. We consider the message in general form $m=\left(m_{i}\right)_{i=1}^{l} \in\{0,1\}^{l}$, and a constant secret key is used. Figure 15 illustrates the dissimilarity matrix for a constant message with the length of $25,100,1000$, and 10 000 bytes. For each constant message, the proposed encoding algorithm is run 100 times. Each time different codewords are generated. Cosine similarity in Kornaropoulos and Efstathopoulos [57] is used to estimate the similarity between two vectors of codewords. As you see, for all cases, the Cosine similarity is less than 0.5. (4) Histogram Analysis. Figures 16(a) and 16(b) show the histogram distribution graphs of a random plaintext with 3000 bytes and transmitted data, respectively. As can be seen from
Figure 16(b), the transmitted data has a balanced histogram distribution and includes all codeword values (0 to 255). Therefore, the proposed method is robust against frequency attack.

5.2. Information Entropy. Information entropy exhibits the discrete probability of random events and can be used to measure system confusion. The ideal value for information entropy should be 8 (Tong et al. [58]). The system information entropy is calculated according to (7), where $P\left(c_{i}\right)$ is the probability of each codeword in the output:

$$
H(c)=\sum_{c} P\left(c_{i}\right) \log _{2} \frac{1}{P\left(c_{i}\right)} .
$$

The information entropy for a given sample message with the lengths of 5000 bytes is 7.9972 . So, the proposed chaos-based encoding algorithm has high performance, because the value of information entropy is close to 8. Also, the information entropy is more close to eight, if the length of data increases. For example, if the message length increases to 100000 bytes, the information entropy is obtained equal to 7.9991. Furthermore, for a given message with all zero bits, information entropy equals 7.7934 (message length $=5000$ bytes). If all bits of the given message are one, entropy is 7.8923 . The information entropy of codewords in these cases is close to 8 . As a result, the proposed scheme generates a set of codewords with more confusion and can resist information entropy attacks well.

The results obtained from observations prove the strength of the scheme against data eavesdropping attacks. Also, the system parameters change adaptively to the message content during running the algorithm, which increases the data security by reducing the time available for statistical attacks on the transmitted codewords. On the other hand, there are about 6000 different sets of codewords to send only one byte of constant data corresponding to the extended SC diagram in Figure 13 (using the same key). Also, there are many different sets of codewords for data with more than six bytes length. As a result, the proposed scheme for secure data transmission is inherently probabilistic and provides high security.

5.3. Comparision. In this section, the proposed scheme is compared with the existing secure data transmission methods in recent years. The comparison is made from four main perspectives: application of chaos in secure data transmission, probabilistic scheme, data type, and keyspace of scheme, as illustrated in Table 2. Chaos-based PRNG is the most common application of chaotic systems in secure data transmission. In Nesa et al. [46], a PRNG is proposed that is based on a logarithmic chaotic map and has been utilized in encrypting sensor data. The authors in Nesa et al. [46] proposed a new deterministic encryption algorithm that generates the same ciphertext for the same plaintext with the same key. As a result, it does not provide a high level of security. In Dhall et al. [45], a random bit insertion phase is employed, which contributes to the probabilistic nature of 


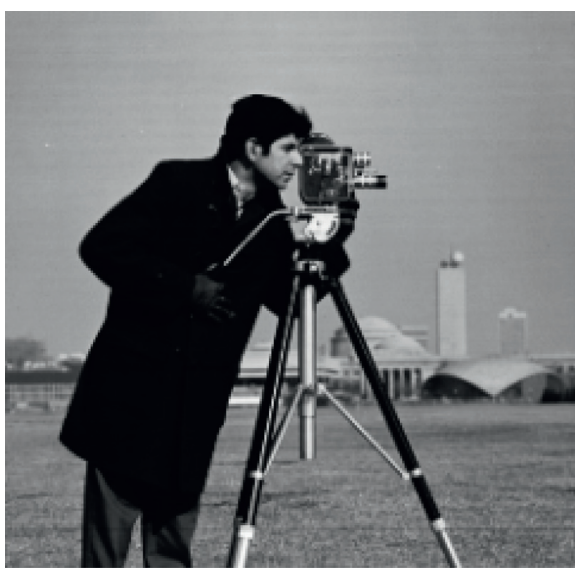

(a)

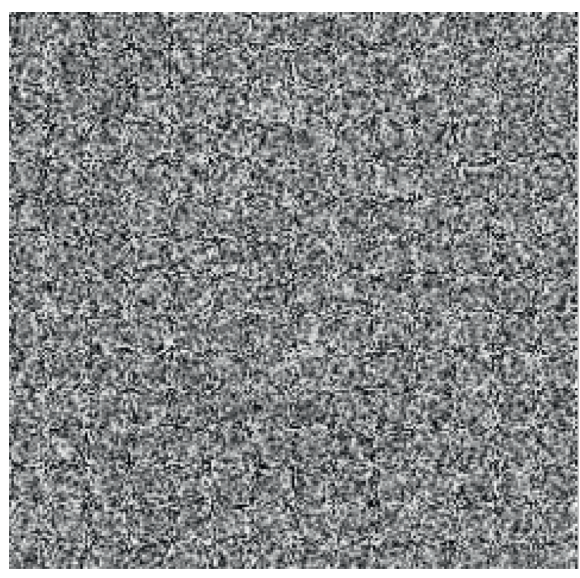

(b)

Figure 14: (a) Original image and (b) extracted image in the receiver with one wrong bit in the secret key.

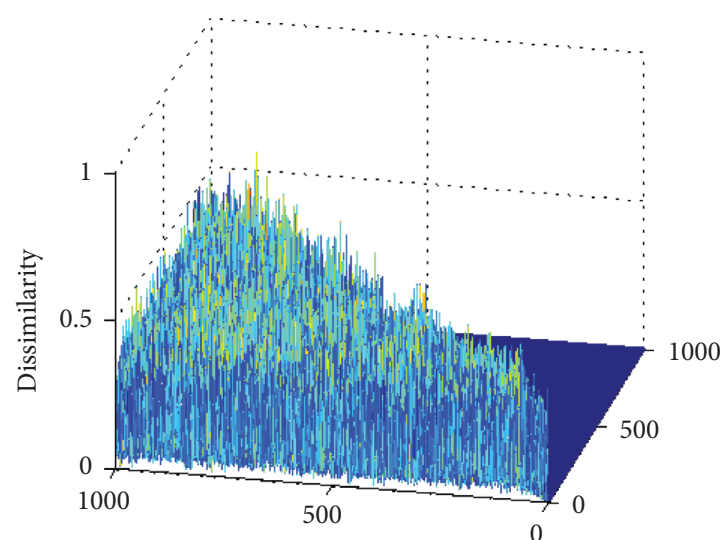

(a)

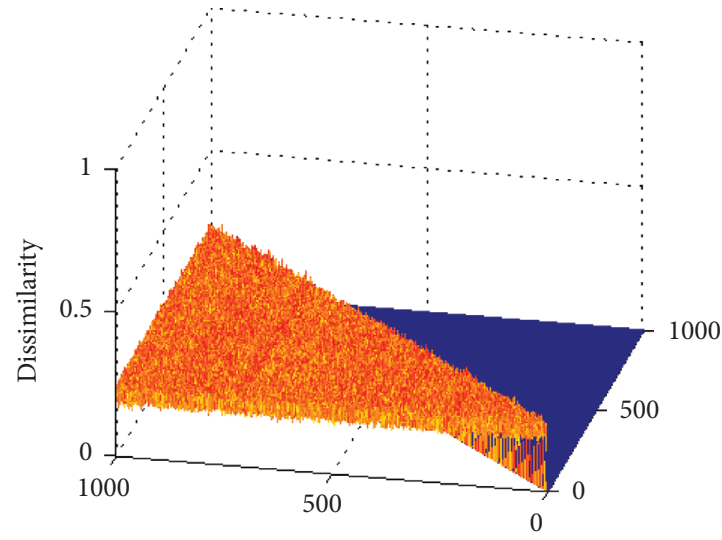

(c)

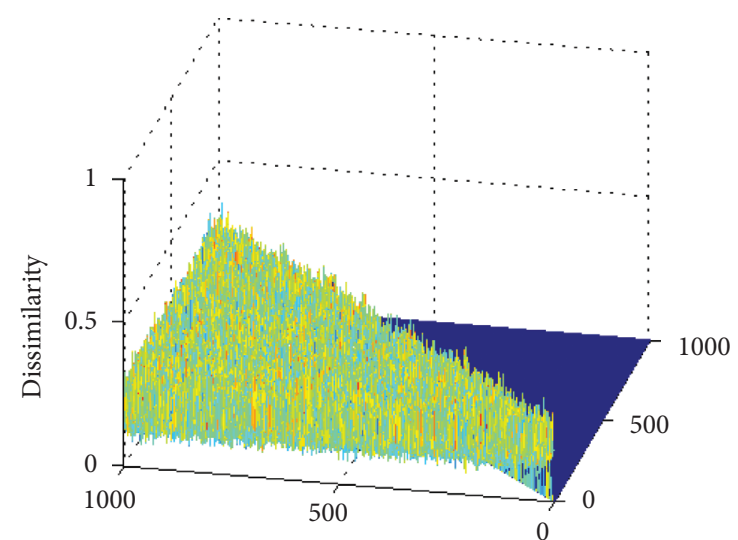

(b)

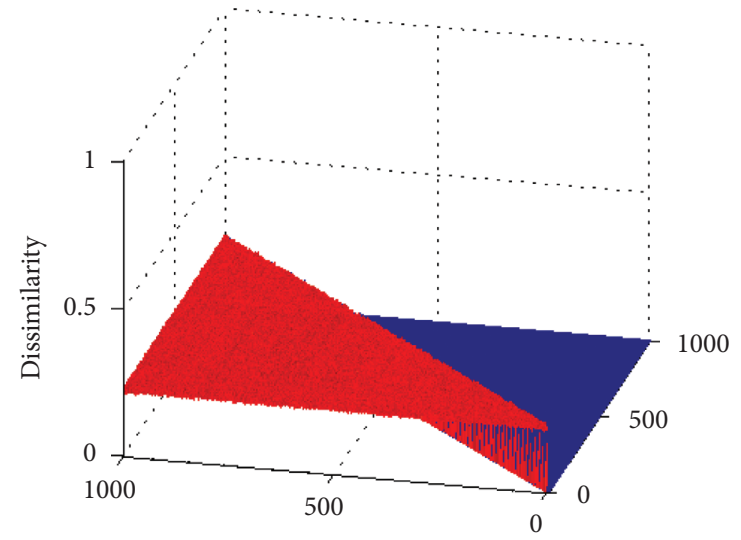

(d)

Figure 15: Dissimilarity matrix for a constant message: (a) message length $=25$ bytes; (b) message length $=100$ bytes; (c) message length $=1000 ;(d)$ message length $=10,000$.

the encryption scheme. The proposed scheme in Dhall et al. [45] uses chaos for generating keystream which is utilized to perform permutation and substitution operations. This probabilistic scheme is suitable for encrypting images. In Srich and San-Um [59], a data encryption scheme is proposed using the chaotic map in order to generate the chaotic matrix. The proposed scheme in Srich and San-Um [59] can also be applied to any data type such as image, text, and excel files. However, it has a deterministic approach and is vulnerable to a chosen-plaintext attack. In this work, we 


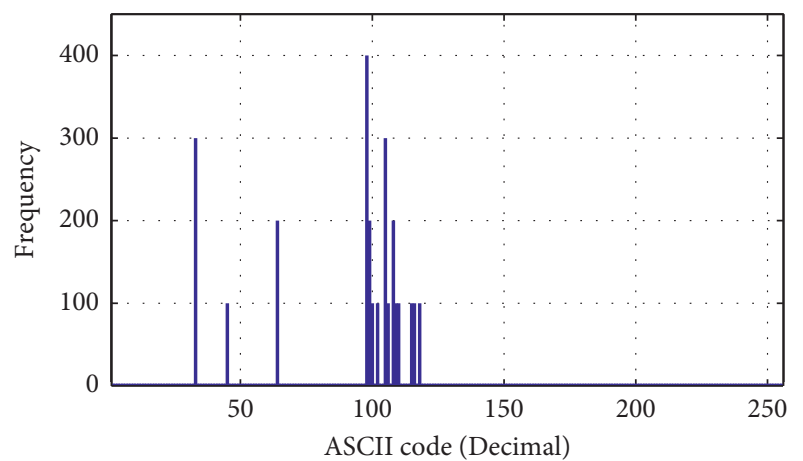

(a)

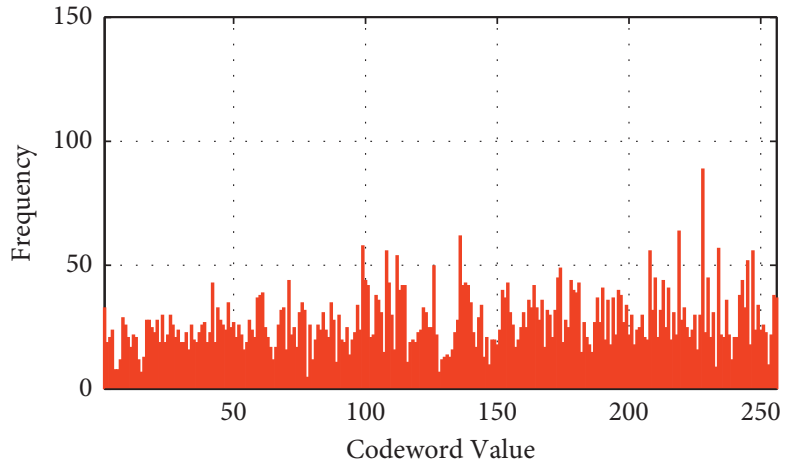

(b)

FIgURE 16: Histogram distribution graph of (a) the plaintext and (b) transmitted data.

TABLE 2: Comparison between the proposed secure data transmission scheme and the other works.

\begin{tabular}{|c|c|c|c|c|}
\hline Scheme & Application of chaos & Probabilistic & Data type & Keyspace \\
\hline Nesa et al. [46] & Chaos-based PRNG & No & $\begin{array}{c}\text { Numeric sensor } \\
\text { data }\end{array}$ & $2^{148}$ \\
\hline Dhall et al. [45] & $\begin{array}{l}\text { Chaos-based permutation and } \\
\text { substitution }\end{array}$ & $\begin{array}{l}\text { Probabilistic with random bits } \\
\text { insertion }\end{array}$ & Image & $2^{256}$ \\
\hline $\begin{array}{l}\text { Srich and San-Um } \\
\text { [59] }\end{array}$ & Chaotic matrix construction & 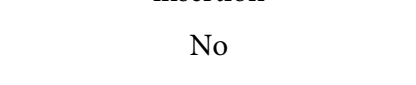 & $\begin{array}{l}\text { Image/Text/Excel } \\
\text { files }\end{array}$ & $\begin{array}{c}2^{(w+3)} 10^{36}(\mathrm{w}: \mathrm{a} \\
\text { parameter })\end{array}$ \\
\hline Proposed scheme & $\begin{array}{l}\text { Chaos-based encoding/decoding } \\
\text { algorithms }\end{array}$ & Inherently probabilistic & $\begin{array}{c}\text { Any type of digital } \\
\text { data }\end{array}$ & $2^{272}$ \\
\hline
\end{tabular}

proposed a new approach for secure data transmission using chaotic systems with multiple coexisting attractors. The proposed chaos-based encoding/decoding algorithms make an evolutionary approach to transmitting confidential data. These algorithms are realized by applying an SC diagram using different steady states. Also, the proposed scheme is inherently probabilistic. The data transmission scheme is flexible since it could be applied to a different type of digital data. The keyspace is large enough to make the attack infeasible in all schemes.

\section{Conclusions}

In this paper, a new non-Shilnikov chaotic system with an infinite number of nonhyperbolic equilibrium points is introduced and analyzed. The novel system has one equilibrium point with triple zero eigenvalues at the origin and many equilibrium points with double zero eigenvalues. Such chaotic systems are very rare in the literature. Also, all equilibrium points are unstable and are located on the $z$-axis. By numerical analysis of the new chaotic system in terms of the phase portrait, bifurcation diagram, Lyapunov exponents, and spectral entropy distribution, a wide variety of dynamics are revealed. The phenomenon of antimonotonicity such as chaotic bubbles and period-2 bubbles is observed in bifurcation diagrams. Also, it is shown that the novel system can exhibit the phenomenon of the infinitely coexistence of multiple periodic, limit cycle, and chaotic attractors. So, the novel chaotic system is multistable. Moreover, the complexity of the system is analyzed by SE.
The SE distribution under different initial values of $y_{0}$ and $z_{0}$ is very scattered, which indicates the coexistence of multiple attractors. Finally, we developed chaos-based encoding/ decoding algorithms for a secure data transmission scheme using the proposed chaotic system. For this purpose, an SC diagram with different steady states is designed. These states are determined by computing the SE complexity of the novel system under different initial values. The proposed secure data transmission scheme is inherently probabilistic and provides high security against different attacks.

As a future task, system (1) will be more investigated by using various functions instead of $\sin (x y-z)$. In a nutshell, the proposed system will be extended and enriched by some proper nonlinearities. More work on constructing many coexisting attractors with non-Shilnikov chaotic systems will be addressed in future research. From an engineering application point of view, we continue to forward the research about secure data transmission schemes via constructing chaotic systems with infinitely equilibrium points. Also, an interesting future direction could be designing the new SC diagrams to improve the efficiency of the secure data transmission scheme.

\section{Data Availability}

In fact, there is not any specific data used in the research work. The authors have solved differential equations in MATLAB. Furthermore, a chaos-based encoding/decoding scheme was applied to the Cameraman image which is well known and could be found everywhere. Overall, there is no data to share with the research community. 


\section{Conflicts of Interest}

The authors declare that they have no conflicts of interest.

\section{References}

[1] J. Sprott, "Do we need more chaos examples?" Chaos Theory and Applications, vol. 2, pp. 49-51, 2020.

[2] J. C. Sprott, "Strange attractors with various equilibrium types," The European Physical Journal-Special Topics, vol. 224, no. 8, pp. 1409-1419, 2015.

[3] S. Zhang, Y. Zeng, Z. Li, M. Wang, X. Zhang, and D. Chang, "A novel simple no-equilibrium chaotic system with complex hidden dynamics," International Journal of Dynamics and Control, vol. 6, no. 4, pp. 1465-1476, 2018.

[4] Q. Deng, C. Wang, and L. Yang, "Four-wing hidden attractors with one stable equilibrium point," International Journal of Bifurcation and Chaos, vol. 30, no. 6, Article ID 2050086, 2020.

[5] V. T. Pham, S. Jafari, X. Wang, and J. Ma, "A chaotic system with different shapes of equilibria," International Journal of Bifurcation and Chaos, vol. 26, Article ID 1650069, 2016.

[6] S. Jafari and J. C. Sprott, "Simple chaotic flows with a line equilibrium," Chaos, Solitons and Fractals, vol. 57, pp. 79-84, 2013.

[7] P. D. K. Kamdem, Q. Lai, and H. Fotsin, "Dynamics, synchronization and electronic implementations of a new lorenzlike chaotic system with nonhyperbolic equilibria," International Journal of Bifurcation and Chaos, vol. 29, no. 14, Article ID 1950197, 2019.

[8] X. Cai, L. Liu, Y. Wang, and C. Liu, “A 3D chaotic system with piece-wise lines shape non-hyperbolic equilibria and its predefined-time control," Chaos, Solitons and Fractals, vol. 146, Article ID 110904, 2021.

[9] L. Chen, F. Nazarimehr, S. Jafari, E. T. Cuautle, and I. Hussain, "Investigation of early warning indexes in a threedimensional chaotic system with zero eigenvalues," Entropy, vol. 22, no. 3, 2020.

[10] C.-L. Li and J.-B. Xiong, "A simple chaotic system with nonhyperbolic equilibria," Optik, vol. 128, pp. 42-49, 2017.

[11] Q. V. Lawande and N. Maiti, "Role of nonlinear dynamics and chaos in applied sciences," Technical Report, Bhabha Atomic Research Centre, Mumbai, Maharashtra, 2000.

[12] S. T. Kingni, L. Keuninckx, P. Woafo, G. Van der Sande, and J. Danckaert, "Dissipative chaos, Shilnikov chaos and bursting oscillations in a three-dimensional autonomous system: theory and electronic implementation," Nonlinear Dynamics, vol. 73, no. 1-2, pp. 1111-1123, 2013.

[13] G. A. Leonov, "Shilnikov chaos in Lorenz-like systems," International Journal of Bifurcation and Chaos, vol. 23, Article ID 1350058, 2013.

[14] E. Izhikevich, "Equilibrium," Scholarpedia, vol. 2, no. 10, 2007.

[15] J. P. Singh and B. K. Roy, "Five new 4-d autonomous conservative chaotic systems with various type of non-hyperbolic and lines of equilibria," Chaos, Solitons and Fractals, vol. 114, pp. 81-91, 2018.

[16] M. Zolfaghari-Nejad, H. Hassanpoor, and M. Charmi, "Numerical analysis of a novel $3 \mathrm{~d}$ chaotic system with periodsubtracting structures," International Journal of Bifurcation and Chaos, vol. 31, no. 11, Article ID 2150169, 2021.

[17] E. Dong, M. Yuan, S. Du, and Z. Chen, "A new class of Hamiltonian conservative chaotic systems with multistability and design of pseudo-random number generator," Applied Mathematical Modelling, vol. 73, pp. 40-71, 2019.
[18] Y. Liu and H. H.-C. Iu, "Antimonotonicity, chaos and multidirectional scroll attractor in autonomous odes chaotic system," IEEE Access, vol. 8, Article ID 77178, 2020.

[19] Y. Li, Y. Zeng, and J. Zeng, "A unique jerk system with abundant dynamics: symmetric or asymmetric bistability, tristability, and coexisting bubbles," Brazilian Journal of Physics, vol. 50, no. 2, pp. 153-163, 2020.

[20] H. Jahanshahi, A. Yousefpour, J. M. P. Munoz, I. Moroz, Z. Wei, and O. Castillo, "A new multi-stable fractional-order four-dimensional system with self-excited and hidden chaotic attractors: dynamic analysis and adaptive synchronization using a novel fuzzy adaptive sliding mode control method," Applied Soft Computing, vol. 87, Article ID 105943, 2020.

[21] S. Jafari and T. Kapitaniak, "Special chaotic systems," The European Physical Journal Special Topics, vol. 229, no. 6-7, pp. 877-886, 2020.

[22] Q. Lai, C. Chen, X. W. Zhao, J. Kengne, and C. Volos, "Constructing chaotic system with multiple coexisting attractors," IEEE Access, vol. 7, Article ID 24056, 2019.

[23] C. Li and J. C. Sprott, "Multistability in the Lorenz system: a broken butterfly," International Journal of Bifurcation and Chaos, vol. 24, no. 10, Article ID 1450131, 2014.

[24] A. N. Pisarchik and C. Grebogi, "Editorial," International Journal of Bifurcation and Chaos, vol. 18, pp. 1605-1606, 2008.

[25] C. Li, W. J. C. Thio, J. C. Sprott, H. H. C. Iu, and Y. Xu, "Constructing infinitely many attractors in a programmable chaotic circuit," IEEE Access, vol. 6, Article ID 29012, 2018.

[26] Q. Lai, P. D. K. Kuate, F. Liu, and H. H. C. Iu, "An extremely simple chaotic system with infinitely many coexisting attractors," IEEE Transactions on Circuits and Systems II: Express Briefs, vol. 67, pp. 1129-1133, 2019.

[27] J. Kengne, A. N. Negou, and D. Tchiotsop, "Antimonotonicity, chaos and multiple attractors in a novel autonomous memristor-based jerk circuit," Nonlinear Dynamics, vol. 88, no. 4, pp. 2589-2608, 2017.

[28] S. T. Kingni, C. Tchodimou, D. F. Platou, P. Djorwe, and S. G. E. Nana, "Antimonotonicity, coexisting attractors and bursting oscillations in optomechanical system: analysis and electronic implementation," The European Physical JournalSpecial Topics, vol. 229, no. 6-7, pp. 1117-1132, 2020.

[29] V. R. F. Signing, J. Kengne, and J. R. M. Pone, "Antimonotonicity, chaos, quasi-periodicity and coexistence of hidden attractors in a new simple 4 -d chaotic system with hyperbolic cosine nonlinearity," Chaos, Solitons and Fractals, vol. 118, pp. 187-198, 2019.

[30] J. Kengne, S. Jafari, Z. T. Njitacke, M. Yousefi Azar Khanian, and A. Cheukem, "Dynamic analysis and electronic circuit implementation of a novel $3 \mathrm{~d}$ autonomous system without linear terms," Communications in Nonlinear Science and Numerical Simulation, vol. 52, pp. 62-76, 2017.

[31] S. Zhang, Y. Zeng, and Z. Li, "Chaos in a novel fractional order system without a linear term," International Journal of NonLinear Mechanics, vol. 106, pp. 1-12, 2018.

[32] Y. Xu and Y. Wang, "A new chaotic system without linear term and its impulsive synchronization," Optik, vol. 125, no. 11, pp. 2526-2530, 2014.

[33] V. T. Pham, S. Jafari, C. Volos, and L. Fortuna, "Simulation and experimental implementation of a line-equilibrium system without linear term," Chaos, Solitons and Fractals, vol. 120, pp. 213-221, 2019.

[34] S. Vaidyanathan, "A novel four-dimensional conservative chaotic system without linear term, its analysis and adaptive control via integral sliding mode control," International 
Journal of Modelling, Identification and Control, vol. 30, no. 2, pp. 132-142, 2018.

[35] V. T. Pham, S. Vaidyanathan, A. T. Azar, and V. H. Duy, "A new chaotic system without linear term, its backstepping control, and circuit design," Backstepping Control of Nonlinear Dynamical Systems, Elsevier, Amsterdam, Netherlands, pp. 33-52, 2021.

[36] S. Mobayen, S. T. Kingni, V.-T. Pham, F. Nazarimehr, and S. Jafari, "Analysis, synchronisation and circuit design of a new highly nonlinear chaotic system," International Journal of Systems Science, vol. 49, no. 3, pp. 617-630, 2018 a.

[37] C. Guanrong, "Chaos theory and applications: a new trend," Chaos Theory and Applications, vol. 3, pp. 1-2, 2021.

[38] Ü Çavuşoğlu, S. Kaçar, I. Pehlivan, and A. Zengin, "Secure image encryption algorithm design using a novel chaos based s-box," Chaos, Solitons and Fractals, vol. 95, pp. 92-101, 2017.

[39] X. Wang, U. Çavuşoğlu, S. Kacar et al., "S-box based image encryption application using a chaotic system without equilibrium," Applied Sciences, vol. 9, no. 4, p. 781, 2019.

[40] E. E. G. García, E. G. Inzunza, O. R. B. López, J. R. V. Cárdenas, and E. T. Cuautle, "Randomness improvement of chaotic maps for image encryption in a wireless communication scheme using pic-microcontroller via zigbee channels," Chaos, Solitons and Fractals, vol. 133, Article ID 109646, 2020.

[41] A. Ullah, A. Javeed, and T. Shah, "A scheme based on algebraic and chaotic structures for the construction of substitution box," Multimedia Tools and Applications, vol. 78, no. 22, Article ID 32484, 2019.

[42] S. Mobayen, C. K. Volos, S. Kaçar, U. Çavuşoğlu, and B. Vaseghi, "A chaotic system with infinite number of equilibria located on an exponential curve and its chaos-based engineering application," International Journal of Bifurcation and Chaos, vol. 28, Article ID 1850112, 2018.

[43] H. Gan, S. Xiao, and Y. Zhao, "A novel secure data transmission scheme using chaotic compressed sensing," IEEE Access, vol. 6, pp. 4587-4598, 2017.

[44] H. Tirandaz and A. K. Mollaee, "On active synchronization of fractional-order Bloch chaotic system and its practical application in secure image transmission," International Journal of Intelligent Computing and Cybernetics, vol. 11, no. 1, 2018.

[45] S. Dhall, S. K. Pal, and K. Sharma, "A chaos-based probabilistic block cipher for image encryption," Journal of King Saud University-Computer and Information Sciences, vol. 34, no. 1, pp. 1533-1543, 2018.

[46] N. Nesa, T. Ghosh, and I. Banerjee, "Design of a chaos-based encryption scheme for sensor data using a novel logarithmic chaotic map," Journal of Information Security and Applications, vol. 47, pp. 320-328, 2019.

[47] S. Shaukat, A. Arshid, A. Eleyan et al., "Chaos theory and its application: an essential framework for image encryption," Chaos Theory and Applications, vol. 2, pp. 17-22, 2020.

[48] H. Noura, C. Guyeux, A. Chehab, M. Mansour, and R. Couturier, "Efficient chaotic encryption scheme with OFB mode," International Journal of Bifurcation and Chaos, vol. 29, Article ID 1950059, 2019.

[49] R. Courtney, Numerical Methods for Solving Systems of Nonlinear Equations, Lakehead University, Thunder Bay, Ontario, Canada, 2012.

[50] J. Jiang, Y. Song, and P. Yu, "Delay-induced triple-zero bifurcation in a delayed leslie-type predator-prey model with additive allee effect," International Journal of Bifurcation and Chaos, vol. 26, Article ID 1650117, 2016.
[51] E. Gamero, E. Freire, A. J. Rodríguez-Luis, E. Ponce, and A. Algaba, "Hypernormal form calculation for triple-zero degeneracies," Bulletin of the Belgian Mathematical Society Simon Stevin, vol. 6, pp. 357-368, 1999.

[52] E. Freire, E. Gamero, A. J. Rodríguez-Luis, and A. Algaba, “A note on the triple-zero linear degeneracy: normal forms, dynamical and bifurcation behaviors of an unfolding," International Journal of Bifurcation and Chaos, vol. 12, no. 12, pp. 2799-2820, 2002.

[53] Z. Faghani, F. Nazarimehr, S. Jafari, and J. C. Sprott, “A new category of three-dimensional chaotic flows with identical eigenvalues," International Journal of Bifurcation and Chaos, vol. 30, Article ID 2050026, 2020.

[54] L. Liu, C. Du, L. Liang, and X. Zhang, "A high spectral entropy (se) memristive hidden chaotic system with multi-type quasiperiodic and its circuit," Entropy, vol. 21, no. 10, 2019.

[55] P. Legendre and L. Legendre, Numerical Ecology, Elsevier, Amsterdam, Netherlands, 2012.

[56] G. Alvarez and S. Li, "Some basic cryptographic requirements for chaos-based cryptosystems," International Journal of Bifurcation and Chaos, vol. 16, pp. 2129-2151, 2006.

[57] E. M. Kornaropoulos and P. Efstathopoulos, "Breaking and fixing secure similarity approximations: dealing with adversarially perturbed inputs," Work, vol. 40, p. 63, 2017.

[58] X. J. Tong, Z. Wang, Y. Liu, M. Zhang, and L. Xu, "A novel compound chaotic block cipher for wireless sensor networks," Communications in Nonlinear Science and Numerical Simulation, vol. 22, no. 1-3, pp. 120-133, 2015.

[59] W. Srich and W. San-Um, "Data encryption scheme based on rules of cellular automata and chaotic map function for information security," IJ Network Security, vol. 18, pp. 11301142, 2016. 